\title{
Dynamics of a Coupled Atmosphere-Ocean Model
}

\author{
Hongjun $\mathrm{Gao}^{1}$ and Jinqiao Duan ${ }^{2}$ \\ 1. Department of Mathematics \\ Nanjing Normal University \\ Nanjing 210097, China \\ and \\ Institute for Mathematics and its Applications \\ University of Minnesota \\ Minneapolis, MN 55455, USA \\ 2. Department of Applied Mathematics \\ Illinois Institute of Technology \\ Chicago, IL 60616, USA \\ E-mail: duan@iit.edu
}

November 23, 2001

\begin{abstract}
The coupled atmosphere-ocean system defines the environment we live. The research of this complex, nonlinear and multiscale system is not only scientifically challenging but also practically important.

We consider a coupled atmosphere-ocean model, which involves hydrodynamics, thermodynamics and nonautonomous interaction at the air-sea interface. First, we show that the coupled atmosphere-ocean system is stable under the external fluctuation in the atmospheric energy balance relation. Then, we estimate the atmospheric temperature feedback in terms of the freshwater flux, heat flux and the external fluctuation at the air-sea interface, as well as the earth's longwave radiation coefficient and the shortwave solar radiation profile. Finally, we prove that the coupled atmosphere-ocean system has time-periodic, quasiperiodic and almost periodic motions, whenever the external fluctuation in the atmospheric energy balance relation is time-periodic, quasiperiodic and almost periodic, respectively.
\end{abstract}

Mathematics Subject Classification:Primary 35K35, 60H15, 76U05; Secondary 86A05, 34D35

Keywords: Nonautonomous dynamical systems, feedback dynamics, attractor, almost periodic motion, geophysical flows, fluctuations

Abbreviated Title: Coupled Atmosphere-Ocean Dynamics 


\section{Introduction: A coupled atmosphere-ocean model}

The global ocean circulation consists of the wind-driven upper ocean circulation and a meridional overturning deep ocean circulation called the thermohaline circulation. The ocean thermohaline circulation involves water masses sinking at high latitudes and upwelling at lower latitudes. During the thermohaline circulation, water masses carry heat or cold around the globe. Thus, it is believed that the global ocean thermohaline circulation plays an important role in the climate [26].

The thermohaline circulation is maintained by water density contrasts in the ocean, which themselves are created by atmospheric forcing, namely, heat and freshwater exchange via evaporation and precipitation at the air-sea interface. Thus the thermohaline circulation is described by coupled atmosphere-ocean models $[24,26]$. Such coupled models also describe feedback of the thermohaline circulation on the atmospheric dynamics (e.g., temperature feedback).

Mathematical models are a key component of our understanding of climate and geophysical systems. The formulation and analysis of mathematical models is central to the progress of better understanding of the thermohaline circulation dynamics and its impact on climate change.

We consider a two-dimensional coupled atmosphere-ocean model, with atmospheric dynamics highly simplified, i.e., the atmospheric dynamics is described by an energy balance model.

This is a zonally averaged, coupled atmosphere-ocean model on the meridional, latitude-depth $(y, z)$-plane as used by various authors $[27,32,4,8,9]$. It is composed of a one-dimensional stochastic energy balance model proposed by North and Cahalan [22], for the latitudinal atmosphere surface temperature $\theta(y, t)$, together with the Boussinesq equations for ocean dynamics in terms of stream function $\psi(y, z, t)$, and transport equations for the oceanic salinity $S(y, z, t)$ and the oceanic temperature $T(y, z, t)$ on the domain $D=\{(y, z): 0 \leq y, z \leq 1\}$ :

$$
\begin{aligned}
\theta_{t}=\theta_{y y}-(a+\theta) & +S_{a}(y)-\gamma(y)\left[S_{o}(y)+\theta-T\right]+f(y, t), 0 \leq z \leq 1 \\
q_{t}+J(q, \psi) & =\operatorname{Pr} \Delta q+\operatorname{Pr} \operatorname{Ra}\left(T_{y}-S_{y}\right), \quad(y, z) \in D \\
T_{t}+J(T, \psi) & =\Delta T, \quad(y, z) \in D \\
S_{t}+J(S, \psi) & =\Delta S, \quad(y, z) \in D
\end{aligned}
$$

where $q(y, z, t)=-\Delta \psi$ is the vorticity; velocity field is $(v, w)=\left(\psi_{z},-\psi_{y}\right) ; a$ is a positive constant parameterizing the effect of the earth's longwave radiative cooling; $S_{a}(y)$ and $S_{o}(y)$ are empirical functions representing the latitudinal dependence of the shortwave solar radiation; $\gamma(y)$ is the latitudinal fraction of the earth covered by the ocean basin; Pr is the Prandtl number and Ra is the Rayleigh number. The first equation is the energy balance model proposed by North and Cahalan [22]. The forcing $f(y, t)$ may arise from, for example, eddy transport fluctuation, stormy bursts of latent heat, and flickering cloudiness variables. Moreover, $J(g, h)=g_{x} h_{y}-g_{y} h_{x}$ is the Jacobian operator and $\Delta=\partial_{y y}+\partial_{z z}$ is the Laplace operator. The effect of the rotation is parameterized in the magnitude of the viscosity and diffusivity terms as discussed in [30]. 
The no-flux boundary condition is taken for the atmosphere temperature $\theta(y, t)$

$$
\theta_{y}(0, t)=\theta_{y}(1, t)=0 \text {. }
$$

The fluid boundary condition is no normal flow and free-slip on the whole boundary

$$
\psi=0, q=0 \text {. }
$$

The flux boundary conditions are assumed for the ocean temperature $T$ and salinity $S$.

At top $z=1$, the fluxes are specified as:

$$
T_{z}=S_{o}(y)+\left.(\theta-T)\right|_{z=1}, \quad S_{z}=F(y),
$$

with $F(y)$ being the given freshwater flux.

At bottom $z=0$ :

$$
T_{z}=S_{z}=0
$$

On the lateral boundary $y=0,1$ :

$$
T_{y}=S_{y}=0 .
$$

We also assume the following compatibility condition:

$$
S_{o}^{\prime}(0)=S_{o}^{\prime}(1)=F^{\prime}(0)=F^{\prime}(1)=0 .
$$

The non-autonomous partial differential equation (1.1) is only defined on the air-sea interface and it may be regarded as a dynamical boundary condition. The boundary condition (1.7) involves a coupling between the atmospheric and oceanic temperature at the air-sea interface.

In the next section, we discuss the well-posedness of this coupled atmosphere-ocean model. Then we investigate the stability of this coupled system under external fluctuation in $\S 3$, atmospheric temperature feedback in $\S 4$, time-periodic, quasiperiodic and almost periodic coupled motion in $\S 5$, respectively. Finally, we summarize these results in the final section $\S 6$.

\section{Mathematical Setup}

In order to use the standard result in [19] for the local existence, we homogenize inhomogeneous boundary conditions for $T, S$ on the top boundary $z=1$ as in [21].

First, we construct two scalar functions:

$$
T_{\epsilon}^{*}=\tilde{T}^{*} \eta_{\epsilon}(z), \quad S_{\epsilon}^{*}=\tilde{S}^{*} \eta_{\epsilon}(z), \forall \epsilon \in\left(0, \frac{1}{2}\right),
$$

where

$$
\begin{aligned}
\tilde{T}^{*} & =\left[S_{o}(y)+\theta\right]\left(1-e^{1-z}\right), \\
\tilde{S}^{*} & =F(y) z, \\
\eta_{\epsilon}(z) & \in C^{\infty}([0,1]) \text { is given by }
\end{aligned}
$$




$$
\eta_{\epsilon}(z)=\left\{\begin{array}{cc}
1, & 1-\epsilon \leq z \leq 1 \\
\text { increasing }, & 1-2 \epsilon \leq z \leq 1-\epsilon \\
0, & 0 \leq z \leq 1-2 \epsilon
\end{array}\right.
$$

Then, set

$$
\hat{T}=T-T_{\epsilon}^{*}, \quad \hat{S}=S-S_{\epsilon}^{*} .
$$

By (1.5) and (1.10), we see that the boundary conditions (1.7) for the new variables $\hat{T}$ and $\hat{S}$ are homogenized and do not affect other boundary conditions. Thus (1.1)-(1.9) become (for the simplicity, we still use $T$ and $S$ instead of $\hat{T}$ and $\hat{S}$ )

$$
\begin{gathered}
\theta_{t}=\theta_{y y}-(a+\theta)+S_{a}(y)-\gamma(y)\left[S_{o}(y)+\theta-T\right]+f(y, t), 0 \leq z \leq 1, \\
q_{t}+J(q, \psi)=\operatorname{Pr} \Delta q+\operatorname{Pr} R a\left(T_{y}-S_{y}+T_{\epsilon y}^{*}-S_{\epsilon y}^{*}\right), \quad(y, z) \in D, \\
T_{t}+J(T, \psi)+J\left(T_{\epsilon}^{*}, \psi\right)=\Delta T+ \\
{\left[\left(\left(1-e^{1-z}\right)(1-\gamma(y))-e^{1-z}\right) \eta_{\epsilon}(z)+\left(1-e^{1-z}\right) \eta_{\epsilon}^{\prime \prime}(z)+2 e^{1-z} \eta_{\epsilon}^{\prime}(z)-e^{1-z} \eta_{\epsilon}\right] \theta} \\
-\left(1-e^{1-z}\right) \eta_{\epsilon}(z) \gamma(y) T(y, 1)+g, \quad(y, z) \in D, \\
S_{t}+J(S, \psi)+J\left(S_{\epsilon}^{*}, \psi\right)=\Delta S \\
+F^{\prime \prime}(y) z \eta_{\epsilon}(z)+F(y)\left(2 \eta_{\epsilon}^{\prime}(z)+z \eta_{\epsilon}^{\prime \prime}(z)\right), \quad(y, z) \in D,
\end{gathered}
$$

where

$$
\begin{aligned}
g(y, z, t)=- & \left(1-e^{1-z}\right) \eta_{\epsilon}(z)\left\{-a+S_{a}(y)-\gamma(y) S_{o}(y)+f(y, t)-S_{o}^{\prime \prime}(y)\right\} \\
& +\left[\left(1-e^{1-z}\right) \eta_{\epsilon}^{\prime \prime}(z)+2 e^{1-z} \eta_{\epsilon}^{\prime}(z)-e^{1-z} \eta_{\epsilon}\right] S_{o}(y) .
\end{aligned}
$$

The corresponding boundary conditions become:

On the whole boundary, the fluid flow satisfies

$$
\psi=0, \quad q=0
$$

The boundary conditions for the atmosphere temperature $\theta(y, t)$ (defined only on the air-sea interface) are

$$
\theta_{y}(0, t)=\theta_{y}(1, t)=0
$$

The boundary conditions for the ocean temperature $T$ and salinity $S$ become At top $z=1$ :

$$
T_{z}+\left.T\right|_{z=1}=0 ; \quad S_{z}=0
$$


At bottom $z=0$ :

$$
T_{z}=S_{z}=0
$$

At the lateral boundary $y=0,1$ :

$$
T_{y}=S_{y}=0 .
$$

The appropriate initial data $\theta_{0}, q_{0}, T_{0}, S_{0}$ are also assumed.

Using the theory in [19], we can obtain the following local existence theorem for problem (2.1)(2.9) (that is (1.1)-(1.9)).

Theorem 2.1 (Local Well-Posedness) Let $\theta_{0} \in H^{1}(0,1), q_{0} \in H_{0}^{1}(D), T_{0}, S_{0} \in H^{1}(D), f \in$ $L^{\infty}\left(0, \infty ; L^{2}(0,1)\right)$. Assume that the physical data satisfy $\gamma(y) \in L^{\infty}(0,1)$, and $S_{o}(y), F(y) \in$ $H^{2}(0,1)$ and aslo assume that the compatibility condition (1.10) be satisfied. Then the coupled atmosphere-ocean system (2.11)-(2.19) (that is (1.1)-(1.9) ) has a unique (The uniqueness of $S$ is up to a constant) local solution satisfying

$$
\begin{gathered}
\theta \in L^{\infty}\left(0, \tau ; H^{1}(0,1)\right) \cap L^{2}\left(0, \tau ; H^{2}(0,1)\right), \\
q \in L^{\infty}\left(0, \tau ; H_{0}^{1}(D)\right) \cap L^{2}\left(0, \tau ; H^{2}(D) \cap H_{0}^{1}(D)\right), \\
T, S \in L^{\infty}\left(0, \tau ; H^{1}(D) \times H^{1}(D)\right) \cap L^{2}\left(0, \tau ; H^{2}(D) \times H^{2}(D)\right),
\end{gathered}
$$

where $\tau$ depends on initial data and physical data $S_{a}(y), S_{o}(y), F(y)$ and $f(y, t)$.

Since $-\Delta \psi=q \in H_{0}^{1}$, we get $\psi \in H_{0}^{1}(D) \cap H^{3}(D)$. Hence the Jacobian $J(\cdot, \cdot)$ is continuous from $H^{1}(D) \times H^{3}(D) \rightarrow L^{2}(D) \times L^{2}(D)$.

In order to obtain the global existence, we need a priori estimates. First, we give a priori estimates for (1.1)-(1.9) in $L^{2}$. In the sequel, $\|\cdot\|$ and $\|\cdot\|_{1}$ denote the norm of $L^{2}$ and $H^{1}$ respectively. Multiplying (1.1) by $\theta$ and performing the integration by parts, we conclude that

$$
\begin{gathered}
\frac{1}{2} \frac{d}{d t}\|\theta\|^{2}=-\left\|\theta_{y}\right\|^{2}-\|\theta\|^{2} \\
-a \int_{0}^{1} \theta d y+\int_{0}^{1} S_{a} \theta d y-\int_{0}^{1} r(y)\left[S_{o}+\theta-T\right] \theta d y+\int_{0}^{1} f \theta d y
\end{gathered}
$$

By the Cauchy-Schwarz inequality, we arrive at

$$
\frac{1}{2} \frac{d}{d t}\|\theta\|^{2} \leq-\left\|\theta_{y}\right\|^{2}+C_{1}\|\theta\|^{2}+\epsilon_{1}\|T(y, 1)\|^{2}+M_{1}
$$

where constant $M_{1}$ depends on $\left\|S_{a}\right\|,\left\|S_{o}\right\|, a$ and $\sup _{0 \leq t<\infty}\|f\|$, constant $C_{1}$ depends on $\|\gamma\|_{L^{\infty}}$ and $\epsilon_{1}>0, \epsilon_{1}>0$ will be chosen later. 
Multiplying (1.2) by $q$, performing the integration by parts and using the property of Jacobian and (1.6), we have

$$
\frac{1}{2} \frac{d}{d t}\|q\|^{2}=-\operatorname{Pr}\|\nabla q\|^{2}+\operatorname{Pr} R a \int_{D}\left(T_{y}-S_{y}\right) q .
$$

Similarly, from (2.13) and (2.14), we have

$$
\frac{1}{2} \frac{d}{d t}\|T\|^{2}=-\|\nabla T\|^{2}+\int_{0}^{1}\left[S_{o}+\theta-T(y, 1)\right] T(y, 1) d y,
$$

and

$$
\frac{1}{2} \frac{d}{d t}\|S\|^{2}=-\|\nabla S\|^{2}+\int_{0}^{1} F(y) S(y, 1) d y
$$

Note that

$$
\begin{aligned}
& \operatorname{PrRa} \int_{\Omega}\left(T_{y}-S_{y}\right) q \leq \frac{\operatorname{Pr}}{2 \lambda_{1}}\|q\|^{2}+\frac{P r R a^{2} \lambda_{1}}{2}\left(\left\|T_{y}\right\|^{2}+\left\|S_{y}\right\|^{2}\right) \\
& \leq \frac{\operatorname{Pr}}{2}\|\nabla q\|^{2}+\operatorname{Pr} R a^{2} \lambda_{1}\left(\|\nabla T\|^{2}+\|\nabla S\|^{2}\right),
\end{aligned}
$$

where $\lambda_{1}$ is a constant in the inequality $\|v\|^{2} \leq \lambda_{1}\|\nabla v\|, v \in H_{0}^{1}$. Thus (2.22) can be rewritten as

$$
\frac{1}{2} \frac{d}{d t}\|q\|^{2} \leq-\frac{\operatorname{Pr}}{2}\|\nabla q\|^{2}+\operatorname{Pr} R a^{2} \lambda_{1}\left(\|\nabla T\|^{2}+\|\nabla S\|^{2}\right) .
$$

Multiplying (2.23) by $2 \operatorname{Pr} R a^{2} \lambda_{1}$ and (2.24) by $2 \operatorname{Pr} R a^{2} \lambda_{1}$, and adding to (2.25), we get

$$
\begin{gathered}
\frac{1}{2} \frac{d}{d t}\left(\|q\|^{2}+2 \operatorname{Pr} R a^{2} \lambda_{1}\left(\|T\|^{2}+\|S\|^{2}\right)\right) \\
\leq-\frac{\operatorname{Pr}}{2}\|\nabla q\|^{2}-\operatorname{PrRa}^{2} \lambda_{1}\left(\|\nabla T\|^{2}+\|\nabla S\|^{2}\right) \\
+2 \operatorname{Pr} R a^{2} \lambda_{1} \int_{0}^{1}\left[S_{o}+\theta-T(y, 1)\right] T(y, 1) d y+2 \operatorname{Pr} R a^{2} \lambda_{1} \int_{0}^{1} F(y) S(y, 1) d y .
\end{gathered}
$$

By the Cauchy-Schwarz inequality and the trace inequality([12]), we have

$$
\begin{aligned}
& \frac{1}{2} \frac{d}{d t}\left(\|q\|^{2}+2 \operatorname{Pr} R a^{2} \lambda_{1}\|T\|^{2}+2 \operatorname{Pr} R a^{2} \lambda_{1}\|S\|^{2}\right) \\
& \leq-\frac{\operatorname{Pr}}{2}\|\nabla q\|^{2}-\operatorname{Pr} R a^{2} \lambda_{1}\left(\|\nabla T\|^{2}+\|\nabla S\|^{2}\right) \\
& +C_{2}\|\theta\|^{2}-\frac{1}{2}\|T(y, 1)\|^{2}+\epsilon_{2}\left(\|\nabla S\|^{2}+\|S\|^{2}\right)+M_{2},
\end{aligned}
$$

where $C_{2}$ depends on $\operatorname{Pr}, R a$ and $\lambda_{1}$, and $M_{2}$ depends on $\operatorname{Pr}, R a, \lambda_{1},\left\|S_{o}\right\|$ and $\|F\|$. Choosing $\epsilon_{1}<\frac{1}{2}$ and $\epsilon_{2}<\frac{\operatorname{Pr} R a^{2} \lambda_{1}}{2}$, combining (2.21) with (2.27), we obtain

$$
\begin{gathered}
\frac{1}{2} \frac{d}{d t}\left(\|\theta\|^{2}+\|q\|^{2}+2 \operatorname{Pr} R a^{2} \lambda_{1}\|T\|^{2}+2 \operatorname{Pr} R a^{2} \lambda_{1}\|S\|^{2}\right) \\
\leq-\alpha\left(\|\nabla \theta\|^{2}+\|\nabla q\|^{2}+\|\nabla T\|^{2}+\|\nabla S\|^{2}\right)+C_{3}\left(\|\theta\|^{2}+\|S\|^{2}\right)+M_{3},
\end{gathered}
$$


where $C_{3}$ depends on $C_{1}$ and $C_{2}, M_{3}$ depends on $M_{1}$ and $M_{2}$, and $\alpha$ is a positive constant depending on $\operatorname{Pr}, R a$ and $\lambda_{1}$. By the Gronwall inequality, we have

$$
\begin{gathered}
\|\theta\|^{2}+\|q\|^{2}+\|T\|^{2}+\|S\|^{2} \\
+\int_{0}^{b}\left(\|\nabla \theta\|^{2}+\|\nabla q\|^{2}+\|\nabla T\|^{2}+\|\nabla S\|^{2}\right) d t \leq C_{1}(b),
\end{gathered}
$$

for any given future time $b(0<b<\infty, 0<t \leq b)$ and some positive constant $C_{1}(b)$ depending on $b, C_{3}$ and $M_{3}$. By a similar argument in [14] (here we need to obtain the estimates in $H^{1} \times$ $H^{1} \times H^{1} \times H^{1} \times H^{1}$ for system of (2.11)-(2.14) in order to avoid the trouble of non-homogeneous boundary conditions, we omit the details here since we will give a similar proof in $\S 4$ ), we have

$$
\begin{gathered}
\|\nabla \theta\|^{2}+\|\nabla q\|^{2}+\|\nabla T\|^{2}+\|\nabla S\|^{2}+ \\
\int_{0}^{b}\left(\|\Delta \theta\|^{2}+\|\Delta q\|^{2}+\|\Delta T\|^{2}+\|\Delta S\|^{2}\right) d t \leq C_{2}(b),
\end{gathered}
$$

for any given $b(0<b<\infty)$ and some positive constant $C_{2}(b)$ depending on $\left\|S_{o}\right\|_{H^{2}},\|F\|_{H^{2}}, b$ and $C_{1}(b)$.

Remark 2.2 In fact, the estimate we get in (2.30) is for $\hat{T}$ and $\hat{S}$, from which we can get the estimate for oringinal $T$ and $S$ using the estimate for $\theta$.

Remark 2.3 Since the equivalence $\|T\|^{2}+\|\Delta T\|^{2}$ with $\|T\|_{H^{2}}^{2}$ and the same for $S$, together with (2.29), we can replace $\int_{0}^{b}\left(\|\Delta \theta\|^{2}+\|\Delta q\|^{2}+\|\Delta T\|^{2}+\|\Delta S\|^{2}\right) d t$ by $\int_{0}^{b}\left(\|\theta\|_{H^{2}}^{2}+\|q\|_{H^{2}}^{2}+\right.$ $\left.\|T\|_{H^{2}}^{2}+\|S\|_{H^{2}}^{2}\right) d t$ in the estimate (2.30).

With these global estimates, we have the following global existence theorem for the coupled atmosphere-ocean system:

Theorem 2.4 (Global Well-Posedness) Let $\theta_{0} \in H^{1}(0,1), q_{0} \in H_{0}^{1}(D), T_{0}, S_{0} \in H^{1}(D), f \in$ $L^{\infty}\left(0, \infty ; L^{2}(0,1)\right)$. Assume that the physical data satisfy $\gamma(y) \in L^{\infty}(0,1)$, and $S_{o}(y), F(y) \in$ $H^{2}(0,1)$ and aslo assume that the compatibility condition (1.10) be satisfied. Then for any given $b(0<b<\infty)$, the coupled atmosphere-ocean system (2.11)-(2.19) (that is (1.1)-(1.9)) has a unique (The uniqueness of $S$ is up to a constant) global solution satisfying

$$
\begin{gathered}
\theta \in L^{\infty}\left(0, b ; H^{1}(0,1)\right) \cap L^{2}\left(0, b ; H^{2}(0,1)\right), \\
q \in L^{\infty}\left(0, b ; H_{0}^{1}(D)\right) \cap L^{2}\left(0, b ; H^{2}(D) \cap H_{0}^{1}(D)\right), \\
T, S \in L^{\infty}\left(0, b ; H^{1}(D) \times H^{1}(D)\right) \cap L^{2}\left(0, b ; H^{2}(D) \times H^{2}(D)\right) .
\end{gathered}
$$

In the rest of this paper, we assume the conditions in this theorem are satisfied, so that we always have global unique solutions.

In the next section, we consider the stability of the above coupled atmosphere-ocean system with respect to the external fluctuation $f(y, t)$ in the atmospheric energy balance dynamics (1.1). 


\section{Stability under External Fluctuation}

Paleo-evidence on the instability of the thermohaline circulation is now abundant. Numerical work suggested that a sufficiently large external forcing (such as external fluctuations in the atmospheric energy balance model and the freshwater flux at the air-sea interface) could destabilize or shutdown the thermohaline circulation [25]. This indicates that current capacity of carrying heat poleward by the thermohaline circulation may change when the freshwater budget is altered. Since the thermohaline circulation's important role in redistributing the heat around the globe, a breakdown or instability of the current thermohaline circulation may lead to dramatic climate change [26]. Because of this relation between the thermohaline circulation and climate change, there is growing interest in its stability or instability. This makes the stability issue of the thermohaline circulation not only of scientific but also of great practical importance.

In this section, we prove the stability of the coupled atmosphere-ocean system with respect to the external fluctuation $f(y, t)$ in the atmospheric energy balance dynamics (1.1), i.e., the continuous dependence of solution on $f(y, t)$ in the space $H^{1}$.

Assume that $\left\{\theta_{1}, q_{1}, T_{1}, S_{1}\right\}$ and $\left\{\theta_{2}, q_{2}, T_{2}, S_{2}\right\}$ are solutions with respect to $f_{1}(y, t)$ and $f_{2}(y, t)$. Let

$$
\bar{\theta}=\theta_{1}-\theta_{2}, \bar{q}=q_{1}-q_{2}, \bar{T}=T_{1}-T_{2}, \bar{S}=S_{1}-S_{2}, \bar{f}=f_{1}-f_{2},
$$

then $\bar{\theta}, \bar{q}, \bar{T}$ and $\bar{S}$ satisfy

$$
\begin{aligned}
\bar{\theta}_{t}=\bar{\theta}_{y y}-\bar{\theta} & -\gamma(y)[\bar{\theta}-\bar{T}]+\bar{f}(y, t), \quad 0 \leq z \leq 1, \\
\bar{q}_{t}+J\left(q_{1}, \psi_{1}\right)-J\left(q_{2}, \psi_{2}\right) & =\operatorname{Pr} \Delta \bar{q}+\operatorname{Pr} R a\left(\bar{T}_{y}-\bar{S}_{y}\right), \quad(y, z) \in D, \\
\bar{T}_{t}+J\left(T_{1}, \psi_{1}\right)-J\left(T_{2}, \psi_{2}\right) & =\Delta \bar{T}, \quad(y, z) \in D \\
\bar{S}_{t}+J\left(S_{1}, \psi_{1}\right)-J\left(S_{2}, \psi_{2}\right) & =\Delta \bar{S}, \quad(y, z) \in D
\end{aligned}
$$

The corresponding boundary conditions are as follows.

On the whole boundary:

$$
\begin{gathered}
\bar{\psi}=0, \bar{\Delta} \psi=\bar{q}=0 . \\
\bar{\theta}_{y}(0, t)=\bar{\theta}_{y}(1, t)=0 .
\end{gathered}
$$

At top $z=1$ :

$$
\bar{T}_{z}+\left.\bar{T}\right|_{z=1}=\bar{\theta} ; \bar{S}_{z}=0
$$

At bottom $z=0$ :

$$
\bar{T}_{z}=\bar{S}_{z}=0
$$

At the lateral boundary $y=0,1$ :

$$
\bar{T}_{y}=\bar{S}_{y}=0 .
$$


Similar to the discussion in $\S 2$ above, we have

$$
\begin{gathered}
\frac{1}{2} \frac{d}{d t}\|\bar{\theta}\|^{2}=-\left\|\bar{\theta}_{y}\right\|^{2}-\|\bar{\theta}\|^{2} \\
-\int_{0}^{1} r(y)|\bar{\theta}|^{2} d y+\int_{0}^{1} \gamma(y) T(y, 1) \bar{\theta} d y+\int_{0}^{1} \bar{f} \bar{\theta} d y \\
\frac{1}{2} \frac{d}{d t}\|\bar{q}\|^{2}+\int_{D}\left(J\left(q_{1}, \psi_{1}\right)-J\left(q_{2}, \psi_{2}\right)\right) \bar{q}=-\operatorname{Pr}\|\nabla \bar{q}\|^{2}+\operatorname{Pr} R a \int_{\Omega}\left(\bar{T}_{y}-\bar{S}_{y}\right) \bar{q}, \\
\frac{1}{2} \frac{d}{d t}\|\bar{T}\|^{2}+\int_{D}\left(J\left(T_{1}, \psi_{1}\right)-J\left(T_{2}, \psi_{2}\right)\right) \bar{T}=-\|\nabla \bar{T}\|^{2}+\int_{0}^{1}[\bar{\theta}-\bar{T}(y, 1)] \bar{T}(y, 1) d y, \\
\frac{1}{2} \frac{d}{d t}\|\bar{S}\|^{2}+\int_{D}\left(J\left(S_{1}, \psi_{1}\right)-J\left(S_{2}, \psi_{2}\right)\right) \bar{S}=-\|\nabla \bar{S}\|^{2} .
\end{gathered}
$$

In order to estimate the terms $\int_{D}\left(J\left(q_{1}, \psi_{1}\right)-J\left(q_{2}, \psi_{2}\right)\right) \bar{q}, \quad \int_{D}\left(J\left(T_{1}, \psi_{1}\right)-J\left(T_{2}, \psi_{2}\right)\right) \bar{T}$ and $\int_{D}\left(J\left(S_{1}, \psi_{1}\right)-J\left(S_{2}, \psi_{2}\right)\right) \bar{S}$, we need the following lemma:

Lemma 3.1 The nonlinear Jacobian operaror $J(u, v)$ has the following property

$$
\begin{gathered}
\left.\| J\left(u_{1}, u_{2}\right)-J\left(v_{1}, v_{2}\right)\right) \| \leq \\
\left(\left\|\nabla u_{1}\right\|+\left\|\nabla u_{2}\right\|+\left\|\nabla v_{1}\right\|+\left\|\nabla v_{2}\right\|\right)\left(\left\|\nabla\left(u_{1}-v_{1}\right)\right\|+\left\|\nabla\left(u_{2}-v_{2}\right)\right\|\right),
\end{gathered}
$$

for every $u_{i}, v_{i} \in H^{1}(D)(i=1,2)$.

The proof of this lemma is in [15].

By Lemma 3.1, we have

$$
\begin{gathered}
\int_{D}\left(J\left(q_{1}, \psi_{1}\right)-J\left(q_{2}, \psi_{2}\right)\right) \bar{q} \leq\left(1+\lambda_{1}\right)^{2}\left(\left\|\nabla q_{1}\right\|+\left\|\nabla q_{2}\right\|\right)\|\nabla \bar{q}\|\|\bar{q}\|, \\
\int_{D}\left(J\left(T_{1}, \psi_{1}\right)-J\left(T_{2}, \psi_{2}\right)\right) \bar{T} \\
\leq\left(\left\|\nabla T_{1}\right\|+\left\|\nabla T_{2}\right\|+\lambda_{1}\left(\left\|\nabla q_{1}\right\|+\left\|\nabla q_{2}\right\|\right)\right)\left(\|\nabla \bar{T}\|+\lambda_{1}\|\nabla \bar{q}\|\right)\|\bar{T}\|,
\end{gathered}
$$

and

$$
\begin{gathered}
\int_{D}\left(J\left(S_{1}, \psi_{1}\right)-J\left(S_{2}, \psi_{2}\right)\right) \bar{S} \\
\leq\left(\left\|\nabla S_{1}\right\|+\left\|\nabla S_{2}\right\|+\lambda_{1}\left(\left\|\nabla q_{1}\right\|+\left\|\nabla q_{2}\right\|\right)\right)\left(\|\nabla \bar{S}\|+\lambda_{1}\|\nabla \bar{q}\|\right)\|\bar{S}\| .
\end{gathered}
$$

Note that

$$
\begin{gathered}
\operatorname{PrRa} \int_{\Omega}\left(\bar{T}_{y}-\bar{S}_{y}\right) \bar{q} \leq \frac{\operatorname{Pr}}{2 \lambda_{1}}\|\bar{q}\|^{2}+\operatorname{Pr} R a^{2} \lambda_{1}\left(\left\|\bar{T}_{y}\right\|^{2}+\left\|\bar{S}_{y}\right\|^{2}\right) \\
\leq \frac{\operatorname{Pr}}{2}\|\nabla \bar{q}\|^{2}+\operatorname{Pr} R a^{2} \lambda_{1}\left(\|\nabla \bar{T}\|^{2}+\|\nabla \bar{S}\|^{2}\right) .
\end{gathered}
$$


Then by a similar argument as in $\S 2$ and using the Cauchy-Schwarz inequality, we conclude that

$$
\begin{aligned}
& \frac{1}{2} \frac{d}{d t}\left(\|\bar{\theta}\|^{2}+\|\bar{q}\|^{2}+2 \operatorname{Pr} R a^{2} \lambda_{1}\|\bar{T}\|^{2}+2 \operatorname{Pr} R a^{2} \lambda_{1}\|\bar{S}\|^{2}\right) \\
& \leq C_{4}\left(\|\bar{\theta}\|^{2}+\|\bar{q}\|^{2}+2 \operatorname{Pr} R a^{2} \lambda_{1}\|\bar{T}\|^{2}+2 \operatorname{Pr} R a^{2} \lambda_{1}\|\bar{S}\|^{2}\right)+\|\bar{f}\|^{2},
\end{aligned}
$$

where $C_{4}$ depends on $\operatorname{Pr}, R a, \lambda_{1},\|\gamma\|_{L^{\infty}}$ as well as the $H^{1}$-norm of $q, T$ and $S$. By the Gronwall inequality, we further have

$$
\|\bar{\theta}\|^{2}+\|\bar{q}\|^{2}+\|\bar{T}\|^{2}+\|\bar{S}\|^{2} \leq C(b)\|\bar{f}\|^{2}
$$

for any given $b(0<b<\infty, 0<t \leq b)$ and some positive constant $C(b)$ depending on $b$ and $C_{4}$. Furthermore, we can obtain the similar estimates for the gradient of $\{\theta, q, T, S\}$, we omit the proof here, as the similar derivation will be done in $\S 5$. Thus the solution differences $\bar{\theta}, \bar{q}, \bar{T}, \bar{S}$ and $\bar{f}$ are bounded when the external fluctuation difference $\bar{f}$ is bounded. So we have the following stability theorem.

Theorem 3.2 (Stability under the external fluctuation) The coupled atmosphere-ocean system (2.11)-(2.14) (that is (1.1)-(1.4)) is stable under the external fluctuation in the atmospheric energy balance model. Namely, the solution of the coupled system depend continuously on the external fluctuation $f$ in $H^{1}$.

\section{Dissipativity and Atmospheric Temperature Feedback}

The ocean and the atmosphere are constantly interacting through the air-sea exchange process. It is expected that the thermohaline circulation could provide feedback to the air temperature. This is a direct impact of the thermohaline circulation on the climate. It is desirable to predict or estimate this feedback.

To this end, let us estimate the air temperature $\theta$ in the mean-square norm, in terms of the freshwater flux $F(y)$, external fluctuation $f(y, t)$ in the energy balance model, the earth's longwave radiative cooling coefficient $a$, and the empirical functions $S_{a}(y)$ and $S_{o}(y)$ representing the latitudinal dependence of the shortwave solar radiation, as well as physical parameters $\operatorname{Pr}$ and $R a$.

We will also show that the system generated by (1.1)-(1.9) is a dissipative system in the sense of [18] or [28] under some conditions, that is all solutions $\{\theta, q, T, S\}$ approach a bounded set (so-called absorbing set) in $H^{1}(0,1) \times H_{0}^{1}(D) \times H^{1}(D) \times H^{1}(D)$ as time goes to $+\infty$. Since

$$
\frac{d}{d t} \int_{\Omega} S d y d z=\int_{0}^{1} F(y) d y=\text { constant. }
$$

For simplicity, we assume that

$$
\int_{0}^{1} F(y) d y=0, \quad \int_{D} S d y d z=0
$$


and

$$
0<\gamma(y) \leq 1 \text { or } 0 \leq \gamma(y)<1
$$

First, we derive a uniform estimate for $\{\theta, q, T, S\}$ in $L^{2}(0,1) \times L^{2}(D) \times L^{2}(D) \times L^{2}(D)$.

Using the standard energy estimate as given in $\S 2$, we get

$$
\begin{gathered}
\frac{1}{2} \frac{d}{d t}\|\theta\|^{2}=-\left\|\theta_{y}\right\|^{2}-\|\theta\|^{2} \\
-a \int_{0}^{1} \theta d y+\int_{0}^{1} S_{a} \theta d y-\int_{0}^{1} r(y)\left[S_{o}+\theta-T(y, 1)\right] \theta d y+\int_{0}^{1} f \theta d y \\
\leq-\left\|\theta_{y}\right\|^{2}-(1-\epsilon)\|\theta\|^{2}-\inf _{y \in[0,1]} \gamma(y)\|\theta\|^{2} \\
+\frac{1}{\epsilon}\left[a^{2}+\left\|S_{o}\right\|^{2}+\left\|S_{a}\right\|^{2}+\|f\|^{2}\right]+\|r(y)\|_{L^{\infty}} \int_{0}^{1}|\theta \| T(y, 1)| d y . \\
\frac{1}{2} \frac{d}{d t}\left(\|q\|^{2}+2 \operatorname{Pr} R a^{2} \lambda_{1}\left(\|T\|^{2}+\|S\|^{2}\right)\right) \leq \\
-\frac{\operatorname{Pr}}{2}\|\nabla q\|^{2}-\operatorname{Pr} P a^{2} \lambda_{1}\left(\|\nabla T\|^{2}+\|\nabla S\|^{2}\right) \\
+2 \operatorname{Pr} R a^{2} \lambda_{1} \int_{0}^{1}\left[S_{o}+\theta-T(y, 1)\right] T(y, 1) d y+2 \operatorname{Pr} R a^{2} \lambda_{1} \int_{0}^{1} F(y) S(y, 1) d y . \\
\leq-\frac{\operatorname{Pr}}{2}\|\nabla q\|^{2}-\operatorname{Pr} P a^{2} \lambda_{1}\left(\|\nabla T\|^{2}+\|\nabla S\|^{2}\right)-2 \operatorname{Pr} R a^{2} \lambda_{1}(1-\epsilon) \int_{0}^{1}|T(y, 1)|^{2} d y \\
+2 \operatorname{Pr} R a^{2} \lambda_{1} \int_{0}^{1}|\theta \| T(y, 1)| d y+\frac{{\operatorname{Pr} R a^{2} \lambda_{1}}_{\epsilon}\left\|S_{o}\right\|^{2}+\frac{\operatorname{Pr}^{2} R a^{4} \lambda_{1}^{2}}{\epsilon_{1}}\|F\|^{2}+\epsilon_{1}\|S(y, 1)\|^{2}}{}
\end{gathered}
$$

here $\epsilon>0$ and $\epsilon_{1}>0$ will be chosen later. By the trace inequlity, we have

$$
\|S(y, 1)\|^{2} \leq C\left(\|\nabla S\|^{2}+\|S\|^{2}\right) \leq C\left(1+\bar{\lambda}_{1}\right)\|\nabla S\|^{2},
$$

where $\bar{\lambda}_{1}$ is the constant in the following Poincare inequality (note that $\int_{D} S d y d z=0$ )

$$
\|S\|^{2} \leq \bar{\lambda}_{1}\|\nabla S\|^{2}
$$

Choosing $\epsilon_{1}=\frac{\operatorname{Pr} R a^{2} \lambda_{1}}{2\left(1+\bar{\lambda}_{1}\right) C}$, then (4.4) can be written as

$$
\begin{aligned}
& \frac{1}{2} \frac{d}{d t}\left(\|q\|^{2}+2 \operatorname{Pr} R a^{2} \lambda_{1}\left(\|T\|^{2}+\|S\|^{2}\right)\right) \leq \\
& -\frac{P r}{2}\|\nabla q\|^{2}-\operatorname{Pr} P a^{2} \lambda_{1}\left(\|\nabla T\|^{2}+\frac{1}{2}\|\nabla S\|^{2}\right)-2 \operatorname{Pr} R a^{2} \lambda_{1}(1-\epsilon) \int_{0}^{1}|T(y, 1)|^{2} d y \\
& +2 \operatorname{Pr} R a^{2} \lambda_{1} \int_{0}^{1}|\theta||T(y, 1)| d y+\frac{\operatorname{PrR}^{2} \lambda_{1}}{\epsilon}\left\|S_{o}\right\|^{2}+2 \operatorname{Pr} R a^{2} \lambda_{1}\left(1+\bar{\lambda}_{1}\right) C\|F\|^{2} .
\end{aligned}
$$


Then, multiplying (4.3) by $2 \operatorname{Pr} R a^{2} \lambda_{1}$ and adding to (4.6), we get

$$
\begin{gathered}
\frac{1}{2} \frac{d}{d t}\left(\|q\|^{2}+2 \operatorname{Pr} R a^{2} \lambda_{1}\left(\|\theta\|^{2}+\|T\|^{2}+\|S\|^{2}\right)\right) \leq \\
-2 \operatorname{Pr} R a^{2} \lambda_{1}\left\|\theta_{y}\right\|^{2}-\frac{\operatorname{Pr}}{2}\|\nabla q\|^{2}-\operatorname{PrPa}^{2} \lambda_{1}\left(\|\nabla T\|^{2}+\frac{1}{2}\|\nabla S\|^{2}\right) \\
-2 \operatorname{Pr} R a^{2} \lambda_{1}\left((1-\epsilon)\|\theta\|^{2}-\inf _{y \in[0,1]} \gamma(y)\|\theta\|^{2}-\left(1+\|\gamma\|_{L^{\infty}}\right) \int_{0}^{1}|T(y, 1) \| \theta| d y+(1-\epsilon) \int_{0}^{1}|T(y, 1)|^{2} d y\right) \\
+\frac{\operatorname{Pr} R a^{2} \lambda_{1}}{2 \epsilon}\left[a^{2}+\frac{5}{4}\left\|S_{o}\right\|^{2}++\left\|S_{a}\right\|^{2}+\|f\|^{2}\right]+2 \operatorname{Pr} \operatorname{Ra}^{2} \lambda_{1}\left(1+\bar{\lambda}_{1}\right) C\|F\|^{2} .
\end{gathered}
$$

By (4.2), when $0 \leq \gamma<1$, we could choose $\epsilon$ such that (since $\inf _{y \in[0,1]} \gamma(y)=0$ now)

$$
4(1-\epsilon)^{2}>\left(1+\|\gamma\|_{L^{\infty}}\right)^{2}, \text { i.e. } \epsilon<\frac{1-\|\gamma\|_{L^{\infty}}}{1+\|\gamma\|_{L^{\infty}}}:=\alpha_{0} .
$$

For example, we choose $\epsilon=\frac{\alpha_{0}}{2}$, then

$$
\begin{aligned}
& -2 \operatorname{Pr} R a^{2} \lambda_{1}\left((1-\epsilon)\|\theta\|-\left(1+\|\gamma\|_{L^{\infty}}\right) \int_{0}^{1}|T(y, 1)||\theta| d y+(1-\epsilon) \int_{0}^{1}|T(y, 1)|^{2} d y\right) \\
& <-\frac{P r R a^{2} \lambda_{1} \alpha_{0}}{2}\left(\|\theta\|^{2}+\|T(y, 1)\|^{2}\right) .
\end{aligned}
$$

If $0<\gamma(y) \leq 1$ as in (4.2), we denote $\inf _{y \in[0,1]} \gamma(y)=\beta_{0}$. Then we take $\epsilon=\frac{\beta_{0}}{6}$ to obtain

$$
\begin{gathered}
-2 \operatorname{PrR} a^{2} \lambda_{1}\left(\left(1-\epsilon+\beta_{0}\right)\|\theta\|-\left(1+\|\gamma\|_{L^{\infty}}\right) \int_{0}^{1}|T(y, 1) \| \theta| d y+(1-\epsilon) \int_{0}^{1}|T(y, 1)|^{2} d y\right) \\
<-\frac{{\operatorname{Pr} R a^{2} \lambda_{1} \beta_{0}}_{6}\left(\|\theta\|^{2}+\|T(y, 1)\|^{2}\right) .}{}
\end{gathered}
$$

So, in the case of $0 \leq \gamma(y)<1$, (4.7) can be written as

$$
\begin{gathered}
\frac{1}{2} \frac{d}{d t}\left(\|q\|^{2}+2 \operatorname{Pr} R a^{2} \lambda_{1}\left(\|\theta\|^{2}+\|T\|^{2}+\|S\|^{2}\right)\right) \leq \\
-2 \operatorname{Pr} R a^{2} \lambda_{1}\left\|\theta_{y}\right\|^{2}-\frac{\operatorname{Pr}}{2}\|\nabla q\|^{2}-\operatorname{Pr} R a^{2} \lambda_{1}\left(\|\nabla T\|^{2}+\frac{1}{2}\|\nabla S\|^{2}\right) \\
-\frac{\operatorname{Pr} R a^{2} \lambda_{1} \alpha_{0}}{2}\left(\|\theta\|^{2}+\|T(y, 1)\|^{2}\right) \\
+\frac{\operatorname{Pr} R a^{2} \lambda_{1}}{\alpha_{0}}\left[a^{2}+\frac{5}{4}\left\|S_{o}\right\|^{2}+\left\|S_{a}\right\|^{2}+\|f\|^{2}\right]+2 \operatorname{Pr} R a^{2} \lambda_{1}\left(1+\bar{\lambda}_{1}\right) C\|F\|^{2} .
\end{gathered}
$$

For $0<\gamma(y)<1$, we will have similar estimate. Since

$$
T^{2}(y, z)-T^{2}(y, 1)=2 \int_{1}^{z} T T_{z} d z
$$


we further have

$$
\|T\|^{2} \leq 2 \int_{0}^{1}|T(y, 1)|^{2} d y+4\|\nabla T\|^{2} .
$$

Using the Poincaré inequlity again for $q$ and letting $\alpha_{1}=\min \left\{\frac{\operatorname{Pr} \lambda_{1}}{4}, \frac{\alpha_{0}}{8}, \frac{1}{8}, \frac{\bar{\lambda}_{1}}{8}\right\}$ and $\beta=\min \left\{\frac{\operatorname{Pr} \lambda_{1}}{4}\right.$, $\left.\frac{\operatorname{Pr} R a^{2} \lambda_{1} \alpha_{0}}{4}, \frac{\operatorname{Pr} R a^{2} \lambda_{1}}{4}\right\}$, the estimate (4.8) becomes

$$
\begin{gathered}
\frac{1}{2} \frac{d}{d t}\left(\|q\|^{2}+2 \operatorname{Pr} R a^{2} \lambda_{1}\left(\|\theta\|^{2}+\|T\|^{2}+\|S\|^{2}\right)\right) \leq \\
-\alpha_{1}\left(\|q\|^{2}+2 \operatorname{Pr} R a^{2} \lambda_{1}\left(\|\theta\|^{2}+\|T\|^{2}+\|S\|^{2}\right)\right. \\
-\beta_{1}\left(\left\|\theta_{y}\right\|^{2}+\|\nabla q\|^{2}+\|\nabla T\|^{2}+\|\nabla S\|^{2}+\|T(y, 1)\|^{2}\right) \\
+\frac{\operatorname{Pr} R a^{2} \lambda_{1}}{\alpha_{0}}\left[a^{2}+\frac{5}{4}\left\|S_{o}\right\|^{2}+\|f\|^{2}\right]+2 \operatorname{Pr} R a^{2} \lambda_{1}\left(1+\bar{\lambda}_{1}\right) C\|F\|^{2} .
\end{gathered}
$$

Using the Gronwall inequality, we finally obtain the mean-square norm estimate for the solution of the coupled atmosphere-ocean model:

$$
\begin{gathered}
\|q\|^{2}+2 \operatorname{Pr} R a^{2} \lambda_{1}\left(\|\theta\|^{2}+\|T\|^{2}+\|S\|^{2}\right) \leq \\
e^{-\alpha_{1} t}\left(\left\|q_{0}\right\|^{2}+2 \operatorname{Pr} R a^{2} \lambda_{1}\left(\left\|\theta_{0}\right\|^{2}+\left\|T_{0}\right\|^{2}+\left\|S_{0}\right\|^{2}\right)\right) \\
+\frac{P r R a^{2} \lambda_{1}}{\alpha_{1}}\left[\frac{1}{\alpha_{0}}\left(a^{2}+\frac{5}{4}\left\|S_{o}\right\|^{2}+\left\|S_{a}\right\|^{2}+\|f\|^{2}\right)+2\left(1+\bar{\lambda}_{1}\right) C\|F\|^{2}\right]
\end{gathered}
$$

In particular, we get the mean-square norm estimate for the atmospheric temperature feedback

$$
\begin{gathered}
\|\theta\|^{2} \leq e^{-\alpha_{1} t}\left(\frac{1}{2 \operatorname{Pr} R a^{2} \lambda_{1}}\left\|q_{0}\right\|^{2}+\left\|\theta_{0}\right\|^{2}+\left\|T_{0}\right\|^{2}+\left\|S_{0}\right\|^{2}\right) \\
+\frac{1}{2 \alpha_{1}}\left[\frac{1}{\alpha_{0}}\left(a^{2}+\frac{5}{4}\left\|S_{o}\right\|^{2}+\left\|S_{a}\right\|^{2}+\|f\|^{2}\right)+2\left(1+\bar{\lambda}_{1}\right) C\|F\|^{2}\right] .
\end{gathered}
$$

This atmospheric temperature feedback estimate is in terms of physical quantities such as the freshwater flux $F(y)$, external fluctuation $f(y, t)$ in the energy balance model, the earth's longwave radiative cooling coefficient $a$, and the empirical functions $S_{a}(y)$ and $S_{o}(y)$ representing the latitudinal dependence of the shortwave solar radiation, as well as physical parameters $\operatorname{Pr}$ and $R a$. Here $\lambda_{1}$ and $\bar{\lambda}_{1}$ are the constants in the Poincare inequality on the domain $D$ in the cases of zero Dirichlet boundary condition and zero mean value, respectively. Moreover, $C$ is a constant depending only on the domain $D, \alpha_{0}=\frac{1-\|\gamma\|_{L \infty}}{1+\|\gamma\|_{L \infty}}$, and $\alpha_{1}=\min \left\{\frac{\operatorname{Pr} \lambda_{1}}{4}, \frac{\alpha_{0}}{8}, \frac{1}{8}, \frac{\bar{\lambda}_{1}}{8}\right\}$.

We can furthermore derive solution estimate in $H^{1}$ norm. To do so, we first get from (4.10)

$$
\int_{t}^{t+1}\left(\left\|\theta_{y}\right\|^{2}+\|\nabla q\|^{2}+\|\nabla T\|^{2}+\|\nabla S\|^{2}+\|T(y, 1)\|^{2}\right) \leq
$$




$$
\begin{gathered}
\frac{1}{\beta_{1}} e^{-\alpha_{0} t}\left(\left\|q_{0}\right\|^{2}+2 \operatorname{Pr} R a^{2} \lambda_{1}\left(\left\|\theta_{0}\right\|^{2}+\left\|T_{0}\right\|^{2}+\left\|S_{0}\right\|^{2}\right)\right) \\
+\frac{2 \operatorname{Pr} R a^{2} \lambda_{1}}{\alpha_{1} \beta_{1}}\left(\frac{1}{\alpha_{0}}\left(a^{2}+\frac{5}{4}\left\|S_{o}\right\|^{2}+\left\|S_{a}\right\|^{2}+\|f\|^{2}\right)+2\left(1+\bar{\lambda}_{1}\right) C\|F\|^{2}\right) .
\end{gathered}
$$

So, let $\left\|q_{0}\right\|^{2}+2 \operatorname{Pr} R a^{2} \lambda_{1}\left(\left\|\theta_{0}\right\|^{2}+\left\|T_{0}\right\|^{2}+\left\|S_{0}\right\|^{2}\right)$ be bounded by some (big) upper bound $R^{2}$ and denote $M^{2}=\frac{\operatorname{Pr} R a^{2} \lambda_{1}}{\alpha_{1}}\left(\frac{1}{\alpha_{0}}\left(a^{2}+\frac{5}{4}\left\|S_{o}\right\|^{2}+\left\|S_{a}\right\|^{2}+\|f\|^{2}\right)+2\left(1+\bar{\lambda}_{1}\right) C\|F\|^{2}\right)$. Then there is a time $t^{*} \geq \frac{2}{\alpha_{1}} \ln \frac{R}{M}$ such that

$$
\|q\|^{2}+2 \operatorname{Pr} R a^{2} \lambda_{1}\left(\|\theta\|^{2}+\|T\|^{2}+\|S\|^{2}\right) \leq 2 M^{2}, t \geq t^{*}
$$

and

$$
\int_{t}^{t+1}\left(\left\|\theta_{y}\right\|^{2}+\|\nabla q\|^{2}+\|\nabla T\|^{2}+\|\nabla S\|^{2}+\|T(y, 1)\|^{2}\right) \leq \frac{3}{\beta_{1}} M^{2}, t \geq t^{*} .
$$

Next, we derive a uniform estimate of gradient of $\{\theta, q, T, S\}$ in $L^{2}(0,1) \times L^{2}(D) \times L^{2}(D) \times$ $L^{2}(D)$. In order to avoid the difficulty caused by the non-homogeneous boundary conditions, we use equations (2.11)-(2.19) instead of (1.1)-(1.9).

Multiplying (2.11)-(2.14) by $-\theta_{y y},-\Delta q,-\Delta T$ and $-\Delta S$ respectively, integrating over $(0,1)$ and $D$, noting that $S^{*} \in H^{2}(D)$ is known and $T^{*}$ is only dependent on $\theta$, we get

$$
\begin{gathered}
\frac{1}{2} \frac{d}{d t}\left\|\theta_{y}\right\|^{2}=-\left\|\theta_{y y}\right\|^{2}-\left\|\theta_{y}\right\|^{2} \\
+a \int_{0}^{1} \theta_{y y} d y-\int_{0}^{1} S_{a} \theta_{y y} d y+\int_{0}^{1} r(y)\left[S_{o}+\theta-T(y, 1)\right] \theta_{y y} d y-\int_{0}^{1} f \theta_{y y} d y \\
\frac{1}{2} \frac{d}{d t}\|\nabla q\|^{2}=-\operatorname{Pr}\|\Delta q\|^{2}+\int_{D} J(q, \psi) \Delta q-\int_{D} \operatorname{Pr} R a\left(T_{y}-S_{y}+T_{y}^{*}-S_{y}^{*}\right) \Delta q \\
\frac{1}{2} \frac{d}{d t}\left(\|\nabla T\|^{2}+\|T(y, 1)\|^{2}\right)=-\|\Delta T\|^{2}+\int_{D} J\left(T+T^{*}, \psi\right) \Delta T \\
-\int_{D}\left[\left(\left(1-e^{1-z}\right)(1-\gamma(y))-e^{1-z}\right) \eta_{\epsilon}(z)+\left(1-e^{1-z}\right) \eta_{\epsilon}^{\prime \prime}(z)+2 e^{1-z} \eta_{\epsilon}^{\prime}(z)-e^{1-z} \eta_{\epsilon}\right] \theta \Delta T \\
+\int_{D}\left(1-e^{1-z}\right) \eta_{\epsilon}(z) \gamma(y) T(y, 1) \Delta T-\int_{D} g \Delta T \\
\frac{1}{2} \frac{d}{d t}\|\nabla S\|^{2}=-\|\Delta S\|^{2}+\int_{D} J\left(S+S^{*}, \psi\right) \Delta S \\
-\int_{D} F^{\prime \prime}(y) z \eta_{\epsilon}(z) \Delta S-\int_{D} F(y)\left(2 \eta_{\epsilon}^{\prime}+z \eta_{\epsilon}^{\prime \prime}(z)\right) \Delta S
\end{gathered}
$$

Note that

$$
a \int_{0}^{1} \theta_{y y} d y-\int_{0}^{1} S_{a} \theta_{y y} d y+\int_{0}^{1} r(y)\left[S_{o}+\theta-T(y, 1)\right] \theta_{y y} d y-\int_{0}^{1} f \theta_{y y} d y
$$




$$
\begin{gathered}
\leq \frac{3 \epsilon}{2}\left\|\theta_{y y}\right\|^{2}+\frac{1}{4 \epsilon}\left[a^{2}+\left\|S_{a}\right\|^{2}+\left\|S_{o}\right\|^{2}+\|f\|^{2}+\|\theta\|^{2}+\|T(y, 1)\|^{2}\right] \\
\quad-\int_{D} \operatorname{Pr} R a\left(T_{y}-S_{y}+T_{y}^{*}-S_{y}^{*}\right) \Delta q \leq \\
\frac{P r}{2}\|\Delta q\|^{2}+\frac{5 P r R a^{2}}{2}\left(\|\nabla T\|^{2}+\|\nabla S\|^{2}+\left\|\theta_{y}\right\|^{2}\right)+\frac{5 P r R a^{2}}{2}\left(\left\|S_{o}^{\prime}\right\|^{2}+\left\|F^{\prime}\right\|^{2}\right), \\
-\int_{D}\left[\left(\left(1-e^{1-z}\right)(1-\gamma(y))-e^{1-z}\right) \eta_{\epsilon}(z)+\left(1-e^{1-z}\right) \eta_{\epsilon}^{\prime \prime}(z)+2 e^{1-z} \eta_{\epsilon}^{\prime}(z)-e^{1-z} \eta_{\epsilon}\right] \theta \Delta T \\
\quad+\int_{D}\left(1-e^{1-z}\right) \eta_{\epsilon}(z) \gamma(y) T(y, 1) \Delta T-\int_{D} g \Delta T \\
\leq \frac{1}{2}\|\Delta T\|^{2}+C\left(a^{2}+\left\|S_{a}\right\|^{2}+\left\|S_{o}\right\|_{H^{2}}^{2}+\|f\|^{2}+\|\theta\|^{2}+\|T(y, 1)\|^{2}\right) . \\
-\int_{D} F^{\prime \prime}(y) z \eta_{\epsilon}(z) \Delta S-\int_{D} F(y)\left(2 \eta_{\epsilon}^{\prime}+z \eta_{\epsilon}^{\prime \prime}(z)\right) \Delta S \leq \frac{1}{2}\|\Delta S\|^{2}+C\|F\|_{H^{2}}^{2}
\end{gathered}
$$

About the estimates of $\int_{D} J(q, \psi) \Delta q, \int_{D} J\left(T+T^{*}, \psi\right) \Delta T$ and $\int_{D} J\left(S+S^{*}, \psi\right) \Delta S$, similar to [14], we have

$$
\begin{gathered}
\int_{D} J(q, \psi) \Delta q \leq C\|\Delta \psi\|\|\nabla q\|\|\Delta q\|=C\|q\|\|\nabla q\|\|\Delta q\|, \\
\int_{D} J\left(T+T^{*}, \psi\right) \Delta T \leq C\|q\|(\|\Delta T\|+\|\nabla T\|+\|T\|)\|\nabla T\|+\|q\|\left(\left\|\theta_{y}\right\|+\left\|S_{o}^{\prime}\right\|\right)\|\Delta T\|, \\
\int_{D} J\left(S+S^{*}, \psi\right) \Delta S \leq C\|q\|(\|\Delta S\|+\|\nabla S\|+\|S\|)\|\nabla S\|+\|q\|\left\|F^{\prime}\right\|\|\Delta S\| .
\end{gathered}
$$

Using the Cauchy-Schwarz inequality, (4.20)-(4.26) and (4.14), when $t \geq t^{*}$, we get

$$
\begin{gathered}
\frac{1}{2} \frac{d}{d t}\left(\left\|\theta_{y}\right\|^{2}+\|\nabla q\|^{2}+3 \operatorname{Pr} R a^{2} \lambda_{1}\left(\|\nabla T\|^{2}+\|T(y, 1)\|^{2}+\|\nabla S\|^{2}\right)\right) \\
\leq C\left(\left\|\theta_{y}\right\|^{2}+\|\nabla q\|^{2}+3 \operatorname{Pr} R a^{2} \lambda_{1}\left(\|\nabla T\|^{2}+\|T(y, 1)\|^{2}+\|\nabla S\|^{2}\right)\right. \\
+C\left(a^{2}+\left\|S_{a}\right\|^{2}+\left\|S_{o}\right\|_{H^{2}}^{2}+\|f\|^{2}+\|F\|_{H^{2}}^{2}\right) .
\end{gathered}
$$

By (4.15) and a uniform Gronwall lemma ([28]), we obtain

$$
\begin{gathered}
\left\|\theta_{y}\right\|^{2}+\|\nabla q\|^{2}+3 \operatorname{Pr} R a^{2} \lambda_{1}\left(\|\nabla T\|^{2}+\|T(y, 1)\|^{2}+\|\nabla S\|^{2}\right) \\
\leq C\left(a^{2}+\left\|S_{a}\right\|^{2}+\left\|S_{o}\right\|_{H^{2}}^{2}+\|f\|^{2}+\|F\|_{H^{2}}^{2}\right) .
\end{gathered}
$$

By (4.14) and (4.28), we know there exists an absorbing sets $\mathcal{B}$ in $H^{1}(0,1) \times H_{0}^{1}(D) \times H^{1}(D) \times$ $H^{1}(D)$ for the solution of (1.1)-(1.9):

$\mathcal{B}=\left\{\{\theta, q, T, S\}:\|\theta\|_{1}^{2}+\|q\|_{1}^{2}+\|T\|_{1}^{2}+\|S\|_{1}^{2} \leq C\left(a^{2}+\left\|S_{a}\right\|^{2}+\left\|S_{o}\right\|_{H^{2}}^{2}+\|f\|^{2}+\|F\|_{H^{2}}^{2}\right)\right\}$,

that is, for every bounded set in $H^{1}(0,1) \times H^{1}(D) \times H^{1}(D) \times H^{1}(D)$, when $t \geq t^{*}+1$, the solution of (1.1)-(1.9) will enter into the $\mathcal{B}$.

We summarize our results in section in the following theorem. 
Theorem 4.1 (Atmospheric temperature feedback and dissipativity) Assume that the freshwater flux $F(y)$ has zero mean as in (4.1) and the ocean basin's latitudinal fraction function $\gamma(y)$ is bounded as in (4.2). Then the coupled atmosphere-ocean system (1.1)-(1.4) has an absorbing set in $H^{1}(0,1) \times H_{0}^{1}(D) \times H^{1}(D) \times H^{1}(D)$ as given in (4.29). More importantly, the atmospheric temperature feedback $\theta(y, t)$ is bounded in mean-square norm in terms of physical quantities such as the freshwater flux $F(y)$, external fluctuation $f(y, t)$ in the energy balance model, the earth's longwave radiative cooling coefficient $a$, and the empirical functions $S_{a}(y)$ and $S_{o}(y)$ representing the latitudinal dependence of the shortwave solar radiation, as well as the Prandtl number Pr and the Rayleigh number Ra as in (4.12).

Remark 4.2 Due to $\frac{d}{d t} \int_{D} \bar{S}=0$, and $\int_{D} \bar{S}_{0}=0$, we obtain $\int_{D} \bar{S}=0$. So, as seen in the discussion of this section, we know that the stability (proved in $\S 3$ ) under the exernal fluctuation is uniform in time $t$ when $0<\gamma(y) \leq 1$ or $0 \leq \gamma(y)<1$.

\section{Strong Contraction and Almost Periodic Atmosphere-Ocean Dy- namics}

In this section, we study the coupled atmosphere-ocean dynamical response to almost periodic (in particular, periodic and quasi-periodic) external fluctuation $f(y, t)$ in the atmospheric energy balance model (1.1). A central question is: Does the coupled atmosphere-ocean system respond almost periodically to almost periodic external fluctuation $f(y, t)$ ?

To answer this question, we need to understand the strong contraction property of the coupled atmosphere-ocean system in the absorbing set $\mathcal{B}$ defined in (4.29). Let $\left\{\theta^{i}, q^{i}, T^{i}, S^{i}\right\}$ be two trajectories corresponding to initial values $\left\{\theta_{0}^{i}, q_{0}^{i}, T_{0}^{i}, S_{0}^{i}\right\} \in \mathcal{B}$ for $i=1,2$. Note that these trajectories remain inside $\mathcal{B}$ as $B$ is a forward invariant set. Their difference

$$
\delta \theta=\theta^{1}-\theta^{2}, \delta q=q^{1}-q^{2}, \delta T=T^{1}-T^{2}, \delta S=S^{1}-S^{2}
$$

satisfy the following equations:

$$
\begin{aligned}
\delta \theta_{t}=\delta \theta_{y y}-\delta \theta & -\gamma(y)[\delta \theta-\delta T], \quad 0 \leq z \leq 1, \\
\delta q_{t}+J\left(q_{1}, \psi_{1}\right)-J\left(q_{2}, \psi_{2}\right) & =\operatorname{Pr} \Delta \delta q+\operatorname{Pr} \operatorname{Ra}\left(\delta T_{y}-\delta S_{y}\right),(y, z) \in D, \\
\delta T_{t}+J\left(T_{1}, \psi_{1}\right)-J\left(T_{2}, \psi_{2}\right) & =\Delta \delta T,(y, z) \in D, \\
\delta S_{t}+J\left(S_{1}, \psi_{1}\right)-J\left(S_{2}, \psi_{2}\right) & =\Delta \delta S,(y, z) \in D,
\end{aligned}
$$

The corresponding boundary conditions are:

$$
\delta \theta_{y}(0, t)=\delta \theta_{y}(1, t)=0
$$


On the whole boundary:

$$
\delta \psi=0, \delta q=0
$$

At top $z=1$ :

$$
\delta T_{z}+\left.\delta T\right|_{z=1}=\delta \theta ; \delta S_{z}=0
$$

At bottom $z=0$ :

$$
\delta T_{z}=\delta S_{z}=0
$$

At the lateral boundary $y=0,1$ :

$$
\delta T_{y}=\delta S_{y}=0
$$

The initial conditions are:

$$
\delta \theta_{0}=\theta_{0}^{1}-\theta_{0}^{2}, \delta q_{0}=q_{0}^{1}-q_{0}^{2}, \delta T_{0}=T_{0}^{1}-T_{0}^{2}, \delta S_{0}=S_{0}^{1}-S_{0}^{2}
$$

where $\delta \theta_{0}=\delta \theta(y, 0), \delta q_{0}=\delta q(y, z, 0), \delta T_{0}=\delta T(y, z, 0)$ and $\delta S_{0}=\delta S(y, z, 0)$.

Using energy estimates as in $\S 3$, we have

$$
\begin{gathered}
\frac{1}{2} \frac{d}{d t}\|\delta \theta\|^{2}=-\left\|\delta \theta_{y}\right\|^{2}-\|\delta \theta\|^{2}-\int_{D} \gamma(y)|\delta \theta|^{2} d y-\int_{D} \gamma(y) \delta \theta \delta T(y, 1) d y \\
\frac{1}{2} \frac{d}{d t}\|\delta q\|^{2}=-\operatorname{Pr}\|\nabla \delta q\|^{2}-\int_{D}\left(J\left(q^{1}, \psi^{1}\right)-J\left(q^{2}, \psi^{2}\right)\right) \delta q-\int_{D} \operatorname{Pr} R a\left(\delta T_{y}-\delta S_{y}\right) \delta q, \\
\frac{1}{2} \frac{d}{d t}\|\delta T\|^{2}=-\|\nabla \delta T\|^{2} \\
+\int_{0}^{1}[\delta \theta-\delta T(y, 1)] \delta T(y, 1) d y-\int_{D}\left(J\left(T^{1}, \psi^{1}\right)-J\left(T^{2}, \psi^{2}\right)\right) \delta T \\
\frac{1}{2} \frac{d}{d t}\|\delta S\|^{2}=-\|\nabla \delta S\|^{2}-\int_{D}\left(J\left(S^{1}, \psi^{1}\right)-J\left(S^{2}, \psi^{2}\right)\right) \delta S
\end{gathered}
$$

Using Lemma 3.1, we imply

$$
\begin{gathered}
-\int_{D}\left(J\left(q^{1}, \psi^{1}\right)-J\left(q^{2}, \psi^{2}\right)\right) \delta q \leq\left\|J\left(q^{1}, \psi^{1}\right)-J\left(q^{2}, \psi^{2}\right)\right\|\|\delta q\| \leq \\
\left(\left\|\nabla q^{1}\right\|+\left\|\nabla q^{2}\right\|+\left\|\nabla \psi^{1}\right\|+\left\|\nabla \psi^{2}\right\|\right)(\|\nabla \delta q\|+\|\nabla \delta \psi\|)\|\delta q\| \\
\leq\left(1+\lambda_{1}\right)^{2} \sqrt{\lambda_{1}}\left(\left\|q^{1}\right\|+\left\|q^{2}\right\|\right)\|\nabla \delta q\|^{2}, \\
-\int_{D}\left(J\left(T^{1}, \psi^{1}\right)-J\left(T^{2}, \psi^{2}\right)\right) \delta T \leq\left\|J\left(T^{1}, \psi^{1}\right)-J\left(T^{2}, \psi^{2}\right)\right\|\|\delta T\| \\
\leq\left(\left\|\nabla T^{1}\right\|+\left\|\nabla T^{2}\right\|+\left\|\nabla \psi^{1}\right\|+\left\|\nabla \psi^{2}\right\|\right)(\|\nabla \delta T\|+\|\nabla \delta \psi\|)\|\delta T\| \\
\leq\left(\left\|\nabla T^{1}\right\|+\left\|\nabla T^{2}\right\|+\lambda_{1}\left(\left\|\nabla q^{1}\right\|+\left\|\nabla q^{2}\right\|\right)\right)\left(\left(\|\nabla \delta T\|+\lambda_{1}\|\nabla \delta q\|\right)\|\delta T\|,\right. \\
-\int_{D}\left(J\left(S^{1}, \psi^{1}\right)-J\left(S^{2}, \psi^{2}\right)\right) \delta S \leq\left\|J\left(S^{1}, \psi^{1}\right)-J\left(S^{2}, \psi^{2}\right)\right\|\|\delta S\|
\end{gathered}
$$




$$
\begin{gathered}
\leq\left(\left\|\nabla S^{1}\right\|+\left\|\nabla S^{2}\right\|+\left\|\nabla \psi^{1}\right\|+\left\|\nabla \psi^{2}\right\|\right)(\|\nabla \delta S\|+\|\nabla \delta \psi\|)\|\delta S\| \\
\leq\left(\left\|\nabla S^{1}\right\|+\left\|\nabla S^{2}\right\|+\lambda_{1}\left(\left\|\nabla q^{1}\right\|+\left\|\nabla q^{2}\right\|\right)\right)\left(\left(\|\nabla \delta S\|+\lambda_{1}\|\nabla \delta q\|\right)\|\delta S\| .\right.
\end{gathered}
$$

Other terms in (5.11)-(5.14) can be estimated as in the proof of the existence of the absorbing set in the last section. So we have

$$
\begin{aligned}
& \frac{1}{2} \frac{d}{d t}\left(\|\delta q\|^{2}+2 \operatorname{Pr} R a^{2} \lambda_{1}\left(\|\delta \theta\|^{2}+\|\delta T\|^{2}+\|\delta S\|^{2}\right)\right) \leq \\
& -\alpha_{1}\left(\|\delta q\|^{2}+2 \operatorname{Pr} R a^{2} \lambda_{1}\left(\|\delta \theta\|^{2}+\|\delta T\|^{2}+\|\delta S\|^{2}\right)\right) \\
& -\beta_{1}\left(\left\|\delta \theta_{y}\right\|^{2}+\|\nabla \delta q\|^{2}+\|\nabla \delta T\|^{2}+\frac{1}{2}\|\nabla \delta S\|^{2}+\|\delta T(y, 1)\|^{2}\right) \\
& +\left(1+\lambda_{1}\right)^{2} \sqrt{\lambda_{1}}\left(\left\|q^{1}\right\|+\left\|q^{2}\right\|\right)\|\nabla \delta q\|^{2}+ \\
& 2 \operatorname{Pr} R a^{2} \lambda_{1}\left(\left\|\nabla T^{1}\right\|+\left\|\nabla T^{2}\right\|+\lambda_{1}\left(\left\|\nabla q^{1}\right\|+\left\|\nabla q^{2}\right\|\right)\right)\left(\left(\|\nabla \delta T\|+\lambda_{1}\|\nabla \delta q\|\right)\|\delta T\|\right. \\
& +2 \operatorname{Pr} R a^{2} \lambda_{1}\left(\left\|\nabla S^{1}\right\|+\left\|\nabla S^{2}\right\|+\lambda_{1}\left(\left\|\nabla q^{1}\right\|+\left\|\nabla q^{2}\right\|\right)\right)\left(\left(\|\nabla \delta S\|+\lambda_{1}\|\nabla \delta q\|\right)\|\delta S\| .\right.
\end{aligned}
$$

Now we assume that $C\left(a^{2}+\left\|S_{a}\right\|^{2}+\left\|S_{o}\right\|_{H^{2}}^{2}+\|f\|^{2}+\|F\|_{H^{2}}^{2}\right)$ is small enough. This is a condition imposed on the physical quantities such as the freshwater flux $F(y)$, external fluctuation $f(y, t)$ in the energy balance model, the earth's longwave radiative cooling coefficient $a$, and the empirical functions $S_{a}(y)$ and $S_{o}(y)$ representing the latitudinal dependence of the shortwave solar radiation.

By the Cauchy-Schwarz inequality, (4.5), (4.9) and (4.29), we get

$$
\begin{gathered}
\frac{1}{2} \frac{d}{d t}\left(\|\delta q\|^{2}+2 \operatorname{Pr} R a^{2} \lambda_{1}\left(\|\delta \theta\|^{2}+\|\delta T\|^{2}+\|\delta S\|^{2}\right)\right) \leq \\
-\alpha_{1}\left(\|\delta q\|^{2}+2 \operatorname{Pr} R a^{2} \lambda_{1}\left(\|\delta \theta\|^{2}+\|\delta T\|^{2}+\|\delta S\|^{2}\right)\right) \\
-\frac{\beta_{1}}{2}\left(\left\|\delta \theta_{y}\right\|^{2}+\|\nabla \delta q\|^{2}+\|\nabla \delta T\|^{2}+\frac{1}{2}\|\nabla \delta S\|^{2}+\|\delta T(y, 1)\|^{2}\right) .
\end{gathered}
$$

By the Gronwall's inequality, we obtain the strong contraction in $L^{2}(0,1) \times L^{2}(D) \times L^{2}(D) \times$ $L^{2}(D)$ for the solution of the coupled atmosphere-ocean system (1.1)-(1.9), that is

$$
\begin{gathered}
\|\delta q\|^{2}+2 \operatorname{Pr} R a^{2} \lambda_{1}\left(\|\delta \theta\|^{2}+\|\delta T\|^{2}+\|\delta S\|^{2}\right) \leq \\
e^{-\alpha_{1} t}\left(\left\|\delta q_{0}\right\|^{2}+2 \operatorname{Pr} R a^{2} \lambda_{1}\left(\left\|\delta \theta_{0}\right\|^{2}+\left\|\delta T_{0}\right\|^{2}+\left\|\delta S_{0}\right\|^{2}\right)\right) .
\end{gathered}
$$

Next, we can show the strong contraction of gradient in $L^{2}(0,1) \times L^{2}(D) \times L^{2}(D) \times L^{2}(D)$. We also need to estimate it using (2.11)-(2.19). Noticing that $S^{*}$ is independent on $\{\theta, q, T, S\}$ and $T^{*}$ is only dependent on $\theta$, we get

$$
\delta \theta_{t}=\delta \theta_{y y}-\delta \theta-\gamma(y)[\delta \theta-\delta T(y, 1)], 0 \leq z \leq 1,
$$




$$
\begin{gathered}
\delta q_{t}=\operatorname{Pr} \Delta \delta q \\
\left.-J\left(q^{1}, \psi^{1}\right)+J\left(q^{2}, \psi^{2}\right)+\operatorname{Pr} R a\left(\delta T_{y}-\delta S_{y}\right)+\delta \theta\left(1-e^{1-z}\right) \eta_{\epsilon}(z)\right),(y, z) \in D, \\
\delta T_{t}=\Delta \delta T-J\left(T^{1}, \psi^{1}\right)+J\left(T^{2}, \psi^{2}\right) \\
-J\left(\left(1-e^{1-z}\right) \eta_{\epsilon}(z) \theta^{1}, \psi^{1}\right)+J\left(\left(1-e^{1-z}\right) \eta_{\epsilon}(z) \theta^{1}, \psi^{2}\right)-J\left(\left(1-e^{1-z}\right) \eta_{\epsilon}(z) S_{o}(y), \delta \psi\right) \\
{\left[\left(\left(1-e^{1-z}\right)(1-\gamma(y))-e^{1-z}\right) \eta_{\epsilon}(z)+\left(1-e^{1-z}\right) \eta_{\epsilon}^{\prime \prime}(z)+2 e^{1-z} \eta_{\epsilon}^{\prime}(z)-e^{1-z} \eta_{\epsilon}\right] \delta \theta} \\
-\left(1-e^{1-z}\right) \eta_{\epsilon}(z) \gamma(y) \delta T(y, 1),(y, z) \in D \\
\delta S_{t}=\Delta \delta S-J\left(S^{1}, \psi^{1}\right)+J\left(S^{2}, \psi^{2}\right)+J\left(S_{\epsilon}^{*}, \delta \psi\right),(y, z) \in D
\end{gathered}
$$

The corresponding boundary conditions are:

$$
\delta \theta_{y}(0, t)=\delta \theta_{y}(1, t)=0 .
$$

On the whole boundary:

$$
\delta \psi=0, \delta \Delta \psi=\delta q=0
$$

At top $z=1$ :

$$
\delta T_{z}+\left.\delta T\right|_{z=1}=0 ; \quad \delta S_{z}=0
$$

At bottom $z=0$ :

$$
\delta T_{z}=\delta S_{z}=0
$$

At the lateral boundary $y=0,1$ :

$$
\delta T_{y}=\delta S_{y}=0
$$

The initial conditions are:

$$
\delta \theta_{0}=\theta_{0}^{1}-\theta_{0}^{2}, \delta q_{0}=q_{0}^{1}-q_{0}^{2}, \delta T_{0}=T_{0}^{1}-T_{0}^{2}+\left(1-e^{1-z}\right) \eta_{\epsilon}(z) \delta \theta_{0}, \delta S_{0}=S_{0}^{1}-S_{0}^{2}
$$

where $\delta \theta_{0}=\delta \theta(y, 0), \delta q_{0}=\delta q(y, z, 0), \delta T_{0}=\delta T(y, z, 0)$ and $\delta S_{0}=\delta S(y, z, 0)$.

Multiplying (5.17)-(5.20) by $-\delta \theta_{y y},-\Delta \delta q,-\Delta \delta T$ and $-\Delta \delta S$ respectively, integrating over $(0,1)$ and $D$, we get

$$
\begin{gathered}
\frac{1}{2} \frac{d}{d t}\left\|\delta \theta_{y}\right\|^{2}=-\left\|\delta \theta_{y y}\right\|^{2}-\left\|\delta \theta_{y}\right\|^{2}+\int_{0}^{1} r(y)[\delta \theta-\delta T(y, 1)] \delta \theta_{y y} d y \\
\frac{1}{2} \frac{d}{d t}\|\nabla \delta q\|^{2}=-\operatorname{Pr}\|\Delta \delta q\|^{2}+\int_{D}\left(J\left(q^{1}, \psi^{1}\right)-J\left(q^{2}, \psi^{2}\right)\right) \Delta \delta q \\
\left.-\operatorname{Pr} R a \int_{D}\left(\delta T_{y}-\delta S_{y}\right) \Delta \delta q-\int_{D}\left(1-e^{1-z}\right) \eta_{\epsilon}(z)\right) \delta \theta \Delta \delta q \\
\frac{1}{2} \frac{d}{d t}\left(\|\nabla \delta T\|^{2}+\|\delta T(y, 1)\|^{2}\right)=-\|\Delta \delta T\|^{2}+\int_{D}\left(J\left(T^{1}, \psi^{1}\right)-J\left(T^{2}, \psi^{2}\right)\right) \Delta \delta T
\end{gathered}
$$




$$
\begin{gathered}
+\int_{D}\left(J\left(\left(1-e^{1-z}\right) \eta_{\epsilon}(z) \theta^{1}, \psi^{1}\right)-J\left(\left(1-e^{1-z}\right) \eta_{\epsilon}(z) \theta^{2}, \psi^{2}\right)\right) \Delta \delta T \\
-\int_{D}\left[\left(\left(1-e^{1-z}\right)(1-\gamma(y))-e^{1-z}\right) \eta_{\epsilon}(z)+\left(1-e^{1-z}\right) \eta_{\epsilon}^{\prime \prime}(z)+2 e^{1-z} \eta_{\epsilon}^{\prime}(z)-e^{1-z} \eta_{\epsilon}\right] \delta \theta \Delta \delta T \\
\quad+\int_{D}\left(1-e^{1-z}\right) \eta_{\epsilon}(z) \gamma(y) \delta T(y, 1) \Delta \delta T \\
+\int_{D} J\left(\left(1-e^{1-z}\right) \eta_{\epsilon}(z) S_{o}(y), \delta \psi\right) \Delta \delta T, \quad(y, z) \in D \\
+\int_{D}\left(J\left(S^{1}, \psi^{1}\right)-J\left(S^{2}, \psi^{2}\right)\right) \Delta \delta S+\int_{D} J\left(S_{\epsilon}^{*}, \delta \psi\right) \Delta \delta S, \quad(y, z) \in D
\end{gathered}
$$

Note that

$$
\begin{gathered}
\int_{0}^{1} r(y)[\delta \theta-\delta T(y, 1)] \delta \theta_{y y} d y \leq \frac{1}{2}\left\|\delta \theta_{y y}\right\|^{2}+\|\gamma(y)\|_{L^{\infty}} \|^{2}\left(\|\delta \theta\|^{2}+\|\delta T(y, 1)\|^{2}\right), \\
-\operatorname{Pr} R a \int_{D}\left(\delta T_{y}-\delta S_{y}\right) \Delta \delta q \leq \frac{P r}{4}\|\Delta \delta q\|^{2}+2 P r R a^{2}\left(\|\nabla \delta T\|^{2}+\|\nabla \delta S\|^{2}\right), \\
\left.-\int_{D}\left(1-e^{1-z}\right) \eta_{\epsilon}(z)\right) \delta \theta \Delta \delta q \leq \frac{P r}{4}\|\Delta \delta q\|^{2}+\frac{1}{P r}\|\delta \theta\|^{2}, \\
-\int_{D}\left[\left(\left(1-e^{1-z}\right)(1-\gamma(y))-e^{1-z}\right) \eta_{\epsilon}(z)+\left(1-e^{1-z}\right) \eta_{\epsilon}^{\prime \prime}(z)+2 e^{1-z} \eta_{\epsilon}^{\prime}(z)-e^{1-z} \eta_{\epsilon}\right] \delta \theta \Delta \delta T \\
\quad \leq \frac{1}{6}\|\Delta T\|^{2}+C\|\delta \theta\|^{2}, \\
\int_{D}\left(1-e^{1-z}\right) \eta_{\epsilon}(z) \gamma(y) \delta T(y, 1) \Delta \delta T \leq \frac{1}{6}\|\Delta \delta T\|^{2}+\frac{3}{2}\|\delta T(y, 1)\|^{2}, \\
\int_{D} J\left(\left(1-e^{1-z}\right) \eta_{\epsilon}(z) S_{o}(y), \delta \psi\right) \Delta \delta T \leq \frac{1}{6}\|\Delta \delta T\|^{2}+C \lambda_{1}\left(\left\|S_{o}\right\|^{2}+\left\|S_{o}^{\prime}\right\|^{2}\right)\|q\|^{2}, \\
\int_{D} J\left(S_{\epsilon}^{*}, \delta \psi\right) \Delta \delta S \leq \frac{1}{2}\|\Delta \delta S\|^{2}+C \lambda_{1}\left(\|F\|^{2}+\left\|F^{\prime}\right\|^{2}\right)\|q\|^{2} .
\end{gathered}
$$

Now by Lemma 3.1, we get

$$
\begin{gathered}
\int_{D}\left(J\left(q^{1}, \psi^{1}\right)-J\left(q^{2}, \psi^{2}\right)\right) \Delta \delta q \leq\|\Delta \delta q\|\left\|J\left(q^{1}, \psi^{1}\right)-J\left(q^{2}, \psi^{2}\right)\right\| \\
\leq \frac{1}{2}\|\Delta \delta q\|^{2}+C\left(1+\lambda_{1}\right)^{2}\|\nabla \delta q\|^{2}, \\
\int_{D}\left(J\left(T^{1}, \psi^{1}\right)-J\left(T^{2}, \psi^{2}\right)\right) \Delta \delta T \leq\|\Delta \delta T\|\left\|J\left(T^{1}, \psi^{1}\right)-J\left(T^{2}, \psi^{2}\right)\right\| \\
\leq \frac{1}{4}\|\Delta \delta T\|^{2}+C\left(\|\nabla \delta q\|^{2}+\mid \nabla \delta T \|^{2}\right),
\end{gathered}
$$




$$
\begin{gathered}
\int_{D}\left(J\left(\left(1-e^{1-z}\right) \eta_{\epsilon}(z) \theta^{1}, \psi^{1}\right)-J\left(\left(1-e^{1-z}\right) \eta_{\epsilon}(z) \theta^{2}, \psi^{2}\right)\right) \Delta \delta T \\
\leq \frac{1}{4}\|\Delta \delta T\|^{2}+C\left(\|\nabla \delta q\|^{2}+\|\nabla \delta \theta\|^{2}\right), \\
\int_{D}\left(J\left(S^{1}, \psi^{1}\right)-J\left(S^{2}, \psi^{2}\right)\right) \Delta \delta S \leq \frac{1}{2}\|\Delta \delta S\|^{2}+C\left(\|\nabla \delta q\|^{2}+\mid \nabla \delta S \|^{2}\right) .
\end{gathered}
$$

Putting the above estimtates together, we conclude

$$
\begin{aligned}
& \frac{1}{2} \frac{d}{d t}\left(\left\|\delta \theta_{y}\right\|^{2}+\|\nabla \delta q\|^{2}+\|\delta T(y, 1)\|^{2}+\|\nabla \delta T\|^{2}+\|\nabla \delta S\|^{2}\right) \\
& \leq C\left(\|\delta \theta\|^{2}+\|\delta T(y, 1)\|^{2}+\|\nabla \delta q\|^{2}+\|\nabla \delta T\|^{2}+\|\nabla \delta S\|^{2}\right) .
\end{aligned}
$$

Taking $N$ large enough, multiplying (5.15) by $N$ and then adding to (5.31), we imply that there exists a positive constant $\alpha_{2}$ such that

$$
\begin{gathered}
\frac{1}{2} \frac{d}{d t}\left(N\|\delta q\|^{2}+2 N \operatorname{Pr} R a^{2} \lambda_{1}\left(\|\delta \theta\|^{2}+\|\delta T\|^{2}+\|\delta S\|^{2}\right)\right) \\
+\frac{1}{2} \frac{d}{d t}\left(\left\|\delta \theta_{y}\right\|^{2}+\|\nabla \delta q\|^{2}+\|\delta T(y, 1)\|^{2}+\|\nabla \delta T\|^{2}+\|\nabla \delta S\|^{2}\right) \\
\leq-\alpha_{2}\left(N\|\delta q\|^{2}+2 N \operatorname{Pr} R a^{2} \lambda_{1}\left(\|\delta \theta\|^{2}+\|\delta T\|^{2}+\|\delta S\|^{2}\right)\right) \\
-\alpha_{2}\left(\left\|\delta \theta_{y}\right\|^{2}+\|\nabla \delta q\|^{2}+\|\nabla \delta T\|^{2}+\frac{1}{2}\|\nabla \delta S\|^{2}+\|\delta T(y, 1)\|^{2}\right) .
\end{gathered}
$$

By the Gronwall inequality, we have

$$
\begin{gathered}
N\|\delta q\|^{2}+2 N \operatorname{Pr} R a^{2} \lambda_{1}\left(\|\delta \theta\|^{2}+\|\delta T\|^{2}+\|\delta S\|^{2}\right) \\
+\left\|\delta \theta_{y}\right\|^{2}+\|\nabla \delta q\|^{2}+\|\delta T(y, 1)\|^{2}+\|\nabla \delta T\|^{2}+\|\nabla \delta S\|^{2} \\
\leq e^{-\alpha_{2} t}\left(N\left\|\delta q_{0}\right\|^{2}+2 N \operatorname{Pr} R a^{2} \lambda_{1}\left(\left\|\delta \theta_{0}\right\|^{2}+\left\|\delta T_{0}\right\|^{2}+\left\|\delta S_{0}\right\|^{2}\right)\right. \\
e^{-\alpha_{2} t}\left(\left\|\delta \theta_{0 y}\right\|^{2}+\left\|\nabla \delta q_{0}\right\|^{2}+\left\|\delta T_{0}(y, 1)\right\|^{2}+\left\|\nabla \delta T_{0}\right\|^{2}+\left\|\nabla \delta S_{0}\right\|^{2}\right) .
\end{gathered}
$$

This estimate tells us that any two solution trajectories inside the absorbing set approach each other as time goes on. This is the so called strong contraction preperty.

Remark 5.1 In fact, here we get the estimate is for $\|\nabla \widehat{\delta T}\|^{2}$ and $\|\nabla \widehat{\delta S}\|^{2}=\|\nabla \delta S\|^{2}$. But $\|\nabla \widehat{\delta T}\|^{2}=\left\|\nabla\left(\delta T+\left(1-e^{1-z}\right) \eta_{\epsilon}(z) \delta \theta\right)\right\|^{2},\|\nabla \widehat{\delta S}\|^{2}=\|\nabla \delta S\|^{2}$ and we have the estimate for $\|\delta \theta\|_{1}$. Hence we can obtain the similar estimate for the original $\delta T$.

Therefore we have the following theorem.

Theorem 5.2 (Strong contraction preperty) Assume that the freshwater flux $F(y)$ has zero mean as in (4.1) and the ocean basin's latitudinal fraction function $\gamma(y)$ is bounded as in (4.2). Let $a^{2}+\left\|S_{a}\right\|^{2}+\left\|S_{o}\right\|_{H^{2}}^{2}+\|f\|^{2}+\|F\|_{H^{2}}^{2}$ be small enough. Then the coupled atmosphere-ocean system (1.1)-(1.4) has the strong contraction property. 
Now we come back to the issue of the time-almost periodic (in particular, time-periodic and timequasi-periodic) motion in the coupled atmosphere-ocean system. First, we give the definitions about almost periodic function and pullback attractor.

A function $\varphi: \mathbb{R} \rightarrow X$, where $\left(X, d_{X}\right)$ is a metric space, is called almost periodic [1] and [29] if for every $\varepsilon>0$ there exists a relatively dense subset $M_{\varepsilon}$ of $\mathbb{R}$ such that

$$
d_{X}(\varphi(t+\tau), \varphi(t))<\varepsilon
$$

for all $t \in \mathbb{R}$ and $\tau \in M_{\varepsilon}$. A subset $M \subseteq \mathbb{R}$ is called relatively dense in $\mathbb{R}$ if there exists a positive number $l \in \mathbb{R}$ such that for every $a \in \mathbb{R}$ the interval $[a, a+l] \cap \mathbb{R}$ of length $l$ contains an element of $M$, i.e. $M \bigcap[a, a+l] \neq \emptyset$ for every $a \in \mathbb{R}$.

In order to study the temporally almost periodic solutions of (1.1)-(1.9), we need some results from the theory of nonautonomous dynamical systems. Consider first an autonomous dynamical system on a metric space $P$ described by a group $\Theta=\left\{\theta_{t}\right\}_{t \in \mathbb{R}}$ of mappings of $P$ into itself.

Let $X$ be a complete metric space and consider a continuous mapping

$$
\Phi: \mathbb{R}^{+} \times P \times X \rightarrow X
$$

satisfying the properties

$$
\Phi(0, p, \cdot)=\mathrm{id}_{X}, \quad \Phi(\tau+t, p, x)=\Phi\left(\tau, \theta_{t} p, \Phi(t, p, x)\right)
$$

for all $t, \tau \in \mathbb{R}^{+}, p \in P$ and $x \in X$. The mapping $\Phi$ is called a cocycle on $X$ with respect to $\Theta$ on $P$.

The appropriate concept of an attractor for a nonautonomous cocycle systems is the pullback attractor. In contrast to autonomous attractors it consists of a family subsets of the original state space $X$ that are indexed by the cocycle parameter set.

A family $\widehat{A}=\left\{A_{p}\right\}_{p \in P}$ of nonempty compact sets of $X$ is called a pullback attractor of the cocycle $\Phi$ on $X$ with respect to $\theta_{t}$ on $P$ if it is $\Phi$-invariant, i.e.

$$
\Phi\left(t, p, A_{p}\right)=A_{\theta_{t}} p \quad \text { for all } \quad t \in \mathbb{R}^{+}, p \in P,
$$

and pullback attracting, i.e.

$$
\lim _{t \rightarrow \infty} H_{X}^{*}\left(\Phi\left(t, \theta_{-t} p, D\right), A_{p}\right)=0 \quad \text { for all } \quad D \in K(X), p \in P,
$$

where $K(X)$ is the space of all nonempty compact subsets of the metric space $\left(X, d_{X}\right)$. Here $H_{X}^{*}$ is the Hausdorff semi-metric between nonempty compact subsets of $X$, i.e. $H_{X}^{*}(A, B):=$ $\max _{a \in A} \operatorname{dist}(a, B)=\max _{a \in A} \min _{b \in B} d_{X}(A, b)$ for $A, B \in K(X)$.

The following theorem combines several known results. See Crauel and Flandoli [7], Flandoli and Schmalfuß [13], and Cheban [2] as well as [20, 6, 3] for this and various related proofs. 
Theorem 5.3 Let $\Phi$ be a continuous cocycle on a metric space $X$ with respect to a group $\Theta$ of continuous mappings on a metric space $P$. In addition, suppose that there is a nonempty compact subset $B$ of $X$ and that for every $D \in K(X)$ there exists $a T(D) \in \mathbb{R}^{+}$, which is independent of $p \in P$, such that

$$
\Phi(t, p, D) \subset B \text { for all } t>T(D) .
$$

Then there exists a unique pullback attractor $\widehat{A}=\left\{A_{p}\right\}_{p \in P}$ of the cocycle $\Phi$ on $X$, where

$$
A_{p}=\bigcap_{\tau \in \mathbb{R}^{+}} \bigcup_{\substack{t>\tau \\ t \in \mathbb{R}^{+}}} \Phi\left(t, \theta_{-t} p, B\right) .
$$

Moreover, if the cocycle $\Phi$ is strongly contracting inside the absorbing set $B$. Then the pullback attractor consists of singleton valued sets, i.e. $A_{p}=\left\{a^{*}(p)\right\}$, and the mapping $p \mapsto a^{*}(p)$ is continuous.

The solution operators $S_{t, t_{0}}$ for (1.1)-(1.9) form a cocycle mapping on $X=H^{1}(0,1) \times H_{0}^{1}(D) \times$ $H^{1}(D) \times H^{1}(D)$ with parameter set $P=\mathbb{R}$, where $p=t_{0}$, the initial time, and $\theta_{t} t_{0}=t_{0}+t$, the left shift by time $t$. However, the space $P=\mathbb{R}$ is not compact here. Though more complicated, it is more useful to consider $P$ to be the closure of the subset $\left\{\theta_{t} f, t \in \mathbb{R}\right\}$, i.e. the hull of $f$, in the metric space $\left.L_{\text {loc }}^{2}(\mathbb{R}, X)\right)$ of locally $L^{2}(\mathbb{R})$-functions $f: \mathbb{R} \rightarrow X$ with the metric

$$
d_{P}(f, g):=\sum_{N=1}^{\infty} 2^{-N} \min \left\{1, \sqrt{\int_{-N}^{N}|\|f(t)-g(t)\||^{2} d t}\right\}
$$

with $\theta_{t}$ defined to be the left shift operator, i.e. $\theta_{t} f(\cdot):=f(\cdot+t)$, where $|\|\cdot\||$ denotes the norm in $X$. By a classical result $[1,29]$, a function $f$ in the above metric space is almost periodic if and only if the the hull of $f$ is compact and minimal. Here minimal means nonempty, closed and invariant with respect to the autonomous dynamical system generated by the shift operators $\theta_{t}$ such that with no proper subset has these properties. The cocycle mapping is defined to be the solution $\vec{\omega}(t)=\{\theta, q, T, S\}$ of (1.1)-(1.9) starting at $\vec{\omega}_{0}=\left\{\theta_{0}, q_{0}, T_{0}, S_{0}\right\} \in X$ at time $t_{0}=0$ for a given forcing mapping $f \in P$, i.e.

$$
\Phi\left(t, f, \omega_{0}\right):=S_{t, 0}^{f} \omega_{0}
$$

where we have included a superscript $f$ on $S$ to denote the dependence on the forcing term $f$. (This dependence is in fact continuous, see $\S 3$ ). The cocycle property here follows from the fact that $S_{t, t_{0}}^{f} \vec{\omega}_{0}=S_{t-t_{0}, 0}^{\theta_{t_{0}} f} \vec{\omega}_{0}$ for all $t \geq t_{0}, t_{0} \in \mathbb{R}, \vec{\omega}_{0} \in X$ and $f \in P$.

Following Theorem 5.3 and the dissipativity and strong contraction properties shown in the last two sections, we conclude that the coupled atmosphere-ocean system (1.1)-(1.4) has a unique pullback attractor, consists of the singleton valued component $\left\{\vec{a}^{*}(p)\right\} \in \hat{A}$ and the mapping $p \mapsto$ 
$\vec{a}^{*}(p)$ is continuous on $P$. As in Duan and Kloeden [10] or Gao, Duan and Fu [16], we now show that this singleton attractor $\vec{a}^{*}(p)$ defines an almost periodic solution.

In fact, the mapping $p \mapsto \vec{a}^{*}(p)$ is uniformly continuous on $P$ because $P$ is compact subset of $\left.L_{l o c}^{2}(\mathbb{R}, X)\right)$ due to the assumed almost periodicity. That is, for every $\varepsilon>0$ there exists a $\delta(\varepsilon)$ $>0$ such that $\left\|\vec{a}^{*}(p)-\vec{a}^{*}(q)\right\|<\varepsilon$ whenever $d_{P}(p, q)<\delta$. Now let the point $\bar{p}(=f$, the given temporal forcing function) be almost periodic and for $\delta=\delta(\varepsilon)>0$ denote by $M_{\delta}$ the relatively dense subset of $\mathbb{R}$ such that $d_{P}\left(\theta_{t+\tau} \bar{p}, \theta_{t} \bar{p}\right)<\delta$ for all $\tau \in M_{\delta}$ and $t \in \mathbb{R}$. From this and the uniform continuity we have

$$
\left\|\vec{a}^{*}\left(\theta_{t+\tau} \bar{p}\right)-\vec{a}^{*}\left(\theta_{t} \bar{p}\right)\right\|<\varepsilon
$$

for all $t \in \mathbb{R}$ and $\tau \in M_{\delta(\varepsilon)}$. Hence $t \mapsto\left\{\theta^{*}, q^{*}, T^{*}, S^{*}\right\}(t):=\vec{a}^{*}\left(\theta_{t} \bar{p}\right)$ is almost periodic, and it is a solution of the coupled atmosphere-ocean model. It is unique as the single-trajectory pullback attractor is the only trajectory that exists and is bounded for the entire time line. Therefore, we have the following result.

Theorem 5.4 (Periodic, quasiperiodic and almost periodic motion) Assume that the freshwater flux $F(y)$ has zero mean as in (4.1) and the ocean basin's latitudinal fraction function $\gamma(y)$ is bounded as in (4.2). Let $a^{2}+\left\|S_{a}\right\|^{2}+\left\|S_{o}\right\|_{H^{2}}^{2}+\|f\|^{2}+\|F\|_{H^{2}}^{2}$ be small enough. Then the coupled atmosphere-ocean system (1.1)-(1.4) has unique time-periodic, quasiperiodic and almost periodic motions, when the external fluctuation $f$ in the atmospheric energy balance model is time-periodic, quasiperiodic and almost periodic, respectively.

\section{Summary}

We have investigated the dynamical behavior of a coupled atmosphere-ocean system.

First, we show that the coupled atmosphere-ocean system is stable under the external fluctuation in the atmospheric energy balance relation (Theorem 3.2). Then, we estimate the atmospheric temperature feedback in terms of the freshwater flux, heat flux and the external fluctuation at the air-sea interface, as well as the earth's longwave radiation and the shortwave solar radiation (Theorem 4.1). Finally, we prove that the coupled atmosphere-ocean system has time-periodic, quasiperiodic and almost periodic motions (under suitable conditions on the physical quantities such as freshwater flux, the earth's longwave radiative cooling coefficient and the shortwave solar radiation profile), when the external fluctuation in the atmospheric energy balance relation is timeperiodic, quasiperiodic and almost periodic, respectively (Theorem 5.4).

Acknowledgement. A part of this work was done at the Oberwolfach Mathematical Research Institute, Germany. This work was partly supported by the NSF Grant DMS-9973204, the Grant 10001018 of the NNSF of China, and the Grant BK2001108 of the NSF of Jiangsu Province, as well as the Scientific Research Foundation for Returned Overseas Chinese Scholars of Jiangsu 
Education Commission. And a part of this work was done while H. Gao was visiting Illinois Institute of Technology and the Institute for Mathematics and Its Applications, USA.

\section{References}

[1] A. Besicovith, Almost Periodic Functions, New York, Dover Publications, 1954.

[2] D. N. Cheban, Global attractors of infinite-dimensional nonautonomous dynamical systems, Izvestiya Akad Nauk RM. Mathematika 25(1997), 42-57.

[3] D. Cheban, P. E. Kloeden and B. Schmalfuss, Pullback attractors in dissipative nonautonomous differential equations under discretization, J. Dyn. Diff. Eqns., 13(2001), 185-213.

[4] F. Chen and M. Ghil, Interdecadal variability in a hybrid coupled ocean-atmosphere model, J. Phys. Oceanography 26 (1996), 1561-1578.

[5] P. Constantin and C. Foias, Navier-Stokes Equations, Univ. of Chicago Press, Chicago, 1988.

[6] H. Crauel, A. Debussche and F. Flandoli, Random attractors, J. Dyn. Diff. Eqns., 9(1997), 307-341.

[7] H. Crauel and F. Flandoli, Attractors for random dynamical systems, Probab. Theory Relat. Fields, 100(1994), 1095-1113.

[8] H. A. Dijkstra and J. D. Neelin, Imperfections of the thermohaline circulation: Latitudinal asymmetry and preferred northern sinking, J. Climate 13 (2000), 366-382.

[9] H. A. Dijkstra, Nonlinear Physical Oceanography, Kluwer Academic Publishers,Boston, 2000.

[10] J. Duan and P. E. Kloeden, Dissipative quasigeostrophic motion under temporally almost periodic forcing, J. Math. Anal. Appl. 236(1999), 74-85.

[11] J. Duan and B. Schmalfuß, The 3D Quasigeostrophic Fluid Dynamics under Random Forcing on Boundary. submitted, 2000.

[12] L. C. Evans, Partial Differential Equations, Amer. Math. Soc., Providence, 1998.

[13] F. Flandoli and B. Schmalfuss, Weak solutions and attractors of the 3D Navier-Stokes equation with nonregular force, Stochastic and Stochastic Reports, 59(1996), 21-45.

[14] H. J. Gao and J. Duan, Dynamics of the thermohaline circulation under wind forcing, Discrite and Continuous Dynamical System B, in press, 2002. 
[15] H. J. Gao and J. Duan, Dynamics of quasi-geostrophic fluid motions with rapidly oscillating Coriolis force, Nonlinear Analysis B, in press, 2002.

[16] H. J. Gao, J. Duan and X. Fu, Almost periodic passive tracer dispersion, J. Math. Anal. Appl. 247(2000), 300-308.

[17] A. E. Gill, Atmosphere-Ocean Dynamics, Academic Press, New York, 1982.

[18] J. K. Hale, Asymptotic Behavior of Dissipative Systems, American Math. Sco., 1988.

[19] D. Henry, Geometric Theory of Semilinear Parabolic Equations, Lectures Notes in Mathematics, Vol 840, Springer-Verlag, New York, 1983.

[20] P. E. Kloeden and B. Schmalfuss, Nonautonomous systems, cocycle attractors and variable time-step discretization, Numer. Algorithms 14(1997), 141-152.

[21] J. L. Lions, R. Temam and S. Wang, On the equations of the large-scale ocean, Nonlinearity 5 (1992), 1007-1053.

[22] G. R. North and R. F. Cahalan, Predictability in a solvable stochastic climate model, J. Atmos. Sci. 38 (1981), 504-513.

[23] J. Pedlosky, Geophysical Fluid Dynamics. Springer Verlag, New-York, Berlin, 1987.

[24] J. P. Peixoto and A. H. Oort, Physics of Climate. Springer, New York, 1992.

[25] S. Rahmstorf, The thermohaline ocean circulation: A system with dangerous thresholds, Climatic Change 46 (2000), 247-256.

[26] G. Siedler, J. Church and J. Gould, Ocean Circulation and Climate: Observing and Modelling the Global Ocean, Academic Press, San Diego, USA, 2001.

[27] T. F. Stocker, D. G. Wright and L. A. Mysak, A zonally averaged, coupled ocean-atmosphere model for paleoclimate studies, J. Climate 5 (1992), 773-797.

[28] R. Temam, Infinite Dimensional Dynamical System in Mechanics and Physics, SpringerVerlag, New-York, 2nd Edition, 1997.

[29] G. R. Sell, Lectures on Topological Dynamics and Differential Equations, Van NostrandReinbold, London, 1971.

[30] O. Thual and J. C. McWilliams, The catastrophe structure of thermohaline convection in a two-dimensional fluid model and a comparison with low-order box model, Geophys. Astrophys. Fluid Dynamics 64 (1992), 67-95. 
[31] W. M. Washington and C. L. Parkinson, An Introduction to Three-Dimensional Climate Modeling, Oxford Univ. Press, 1986.

[32] D. G. Wright and T. F. Stocker, A zonally averaged ocean model for the thermohaline circulation. Part I: Model development and flow dynamics, J. Phys. Oceanography 21 (1991), 1713-1724. 